\title{
TLR3 Mediates Repair and Regeneration of Damaged Neonatal Heart through Glycolysis Dependent YAP1 Regulated miR-152 Expression
}

\author{
Xiaohui Wang ${ }^{1,2} \cdot$ Tuanzhu Ha ${ }^{1,2} \cdot \mathrm{Li} \mathrm{Liu}^{3} \cdot$ Yuanping Hu $\mathrm{Hu}^{1,5} \cdot$ Race Kao $^{1,2} \cdot$ John Kalbfleisch ${ }^{2,4} \cdot$ David Williams $^{1,2}$. \\ Chuanfu $\mathrm{Li}^{1,2}$
}

Received: 18 May 2017 / Revised: 16 October 2017 / Accepted: 3 November 2017 / Published online: 22 January 2018

(c) The Author(s) 2018. This article is published with open access

\begin{abstract}
The present study investigated whether TLR3 is required for neonatal heart repair and regeneration following myocardial infarction (MI). TLR3 deficient neonatal mice exhibited impaired cardiac functional recovery and a larger infarct size, while wild type neonatal mice showed cardiac functional recovery and small infarct size after MI. The data suggest that TLR3 is essential for the regeneration and repair of damaged neonatal myocardium. In vitro treatment of neonatal cardiomyocytes with a TLR3 ligand, Poly (I:C), significantly enhances glycolytic metabolism, YAP1 activation and proliferation of cardiomyocytes which were prevented by a glycolysis inhibitor, 2-deoxyglucose (2-DG). Administration of 2-DG to neonatal mice abolished cardiac functional recovery and YAP activation after MI, suggesting that TLR3-mediated regeneration and repair of the damaged neonatal myocardium is through glycolytic-dependent YAP1 activation. Inhibition of YAP1 activation abolished Poly (I:C) induced proliferation of neonatal cardiomyocytes. Interestingly, activation of YAP1 increases the expression of miR-152 which represses the expression of cell cycle inhibitory proteins, P27kip1 and DNMT1, leading to cardiomyocyte proliferation. We conclude that TLR3 is required for neonatal heart regeneration and repair after MI. The mechanisms involve glycolytic-dependent YAP1 activation, resulting in miR-152 expression which targets DNMT1/p27kip1.
\end{abstract}

\section{Introduction}

Ischemic heart disease remains the major cause of death in the United States [1]. The adult heart has limited capacity to

\section{Edited by D. Aberdam}

Electronic supplementary material The online version of this article (https://doi.org/10.1038/s41418-017-0036-9) contains supplementary material, which is available to authorized users.

$\triangle$ Chuanfu Li

Li@etsu.EdU

1 Departments of Surgery, East Tennessee State University, Johnson City, TN, USA

2 Center of Excellence in Inflammation, Infectious Disease and Immunity, James H. Quillen College of Medicine, East Tennessee State University, Johnson City, TN, USA

3 Department of Geriatrics, The First Affiliated Hospital of Nanjing Medical University, Nanjing, China

4 Biometry and Medical Computing and East Tennessee State University, Johnson City, TN, USA

5 Present address: Department of Pharmacy, the Binhu Hospital of Hefei, Anhui, China regenerate and repair damaged myocardium induced by ischemia/reperfusion (I/R) injury. Interestingly, the hearts of zebrafish and the neonatal mouse have the ability to repair and regenerate damaged myocardium [2-4]. However, neonatal mouse hearts lose the capacity for proliferation and regeneration 7 days after birth [2], which positively correlates with the changes in cardiomyocyte metabolism from glycolysis to oxidative phosphorylation. It is well known that $>90 \%$ of the energy in adult cardiomyocytes is generated by mitochondrial oxidative phosphorylation [5], suggesting that glycolysis could play an important role in the proliferation of cardiomyocytes. Indeed, glycolytic metabolism is predominant in zebrafish and neonatal cardiomyocytes $[6,7]$ and is essential for somatic cell reprogramming and differentiation [8-10]. Therefore, enhanced glycolytic metabolism could be an important approach for induction of cardiomyocyte proliferation.

Toll-like receptors (TLRs) are the pattern-recognition receptors that play a critical role in the induction of innate immune and inflammatory responses $[11,12]$. TLR ligands can promote metabolic reprogramming from oxidative phosphorylation to glycolysis which is necessary for activation of immune cells and for trained innate immunity [13-15]. However, the mechanisms remain elusive. 
YAP and TAZ are major downstream effectors of the Hippo signaling pathway which play critical roles in controlling organ size [16]. The Hippo pathway is comprised of core kinase complexes including mammalian STE20-like protein kinase 1 and 2 (MST1/2), large tumor suppressor 1 and 2 (LATS1/2), and the adaptor proteins SAV1 and MOB1. Activation of MST1/2 phosphorylates LATS1/2 which suppresses YAP/TAZ transcriptional activity by phosphorylation [16, 17]. YAP1 and TAZ have been demonstrated to regulate cardiomyocyte proliferation and regeneration [17-22].

In the present study, we demonstrated that TLR3 is required for neonatal damaged heart regeneration. TLR3 activation induces glycolysis dependent YAP1 activation which regulates the expression of microRNA-152 to target cell cycle inhibitory proteins DNMT1/p27kip1, leading to regulation of neonatal cardiomyocyte proliferation.

\section{Results}

\section{TLR3 Deficiency Impairs Neonatal Heart Regeneration After MI}

To investigate whether TLR3 could be involved in neonatal heart regeneration and repair, 1 day old wild type (WT) or TLR3 deficient $\left(\right.$ TLR3 $^{-/}$) mice were subjected to myocardial infarction (MI). Cardiac function was examined by echocardiography and cardiomyocyte proliferation was evaluated by 5-ethynyl-2'-deoxyuridine (EdU) incorporation 21 days after MI. As shown in Fig. 1a, WT neonatal mice show a smaller infarct size and smaller fibrotic area, while TLR3 ${ }^{-1-}$ neonatal hearts exhibit larger scarring and increased fibrotic deposition, when compared with WT MI mice. Fig. 1b shows that EdU positive staining of cardiomyocytes in WT neonatal MI heart tissues is significantly greater $(52.9 \%)$ than that in

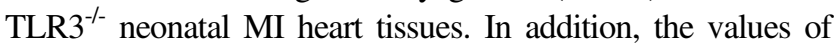
cardiac function in WT neonatal MI mice are compatible with WT sham control (Fig. 1c). In contrast, the values of cardiac function in $\mathrm{TLR}^{-/-}$neonatal MI mice are significantly lower than that in $\mathrm{TLR}^{-/-}$sham control and WT MI mice (Fig. 1c). The data suggest that TLR3 is necessary for neonatal heart regeneration and repair after $\mathrm{MI}$.

\section{TLR3 Ligand, Poly (I:C) Enhances Glycolysis And Promotes Cardiomyocyte Proliferation}

To investigate whether the TLR3 ligand, Poly (I:C) would enhance glycolysis in cardiomyocytes, we isolated neonatal cardiomyocytes from 1 day old WT mice, treated them with Poly (I:C) for $12 \mathrm{~h}$, and analyzed glycolytic metabolism. As shown in Fig. 1d, Poly (I:C) treatment markedly enhances glycolysis and glycolytic capacity and significantly increases the proliferation rate of neonatal cardiomyocytes as evidenced by increased EdU staining of neonatal cardiomyocytes (Fig. 1e), when compared with untreated controls. Interestingly, treatment of neonatal cardiomyocytes with 2-deoxy-D-glucose (2-DG), an inhibitor for hexokinase 2 which is an initial step in glycolysis, prevents Poly (I:C) induced neonatal cardiomyocyte proliferation (Fig. 1e). Importantly, in vivo administration of 2-DG to 1 day old (P1) WT neonatal mice immediately after induction of MI significantly impairs cardiac functional recovery (Fig. 1f). The data suggest that glycolytic metabolism plays a critical role in TLR3-mediated neonatal cardiomyocyte proliferation and neonatal heart regeneration and repair after MI.

\section{TLR3 Mediates YAP1 Activation via a Glycolytic- Dependent Mechanism}

To address how enhanced glycolysis by Poly (I:C) promotes neonatal cardiomyocyte proliferation, we investigated whether YAP1/TAZ activation involves TLR3-mediated neonatal heart regeneration and repair following $\mathrm{MI}$. Fig. 2a, b show that the levels of YAP1/TAZ in cytosol and nuclei were markedly increased in WT MI hearts compared with WT sham control. In TLR3 ${ }^{-/}$MI mice, however, the levels of YAP1/TAZ in the cytosol and nuclei did not significantly change compared with $\mathrm{TLR}^{-/-}$sham control and were markedly lower than that in WT MI mice. The data suggest that TLR3 is essential for YAP1/TAZ activation after MI.

We then examined whether Poly (I:C) induced activation of YAP1 is mediated by glycolysis. As shown in Fig. 2c, d, Poly (I:C) treatment significantly increases the levels of YAP1 in both cytosol and nuclei in WT cardiomyocytes, but not in $\mathrm{TLR}^{-/}$cardiomyocytes. However, inhibition of glycolysis by 2-DG prevented Poly I:C-induced activation of YAP1 (Fig. 2e, f), suggesting that TLR3-mediated YAP1 activation is through glycolytic-dependent mechanism.

\section{YAP1 activation is required for TLR3-mediated cardiomyocyte proliferation}

To determine the role of YAP/TAZ in TLR3-mediated cardiomyocyte proliferation, we analyzed the expression pattern of YAP/TAZ in the myocardium at the different stages of neonatal heart maturation. As shown in Fig. 3a, the levels of YAP1/TAZ expression are the highest in P1 and P3 neonatal hearts, gradually reduced on P7, and remained low on $\mathrm{P} 14$, and $\mathrm{p} 21$ which positively correlated with a loss of regenerative capacity in damaged neonatal hearts. To confirm that YAP1 plays an important role in cardiomyocyte proliferation, we transfected neonatal cardi-

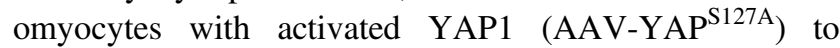
induce overexpression of YAP1. AAV-Luci served as a 
A

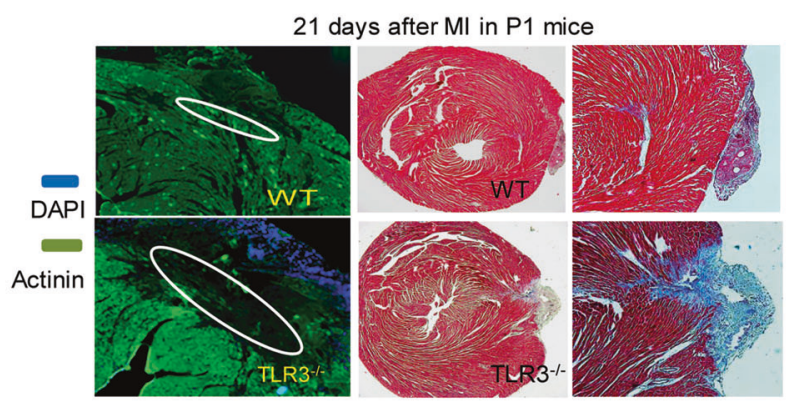

B

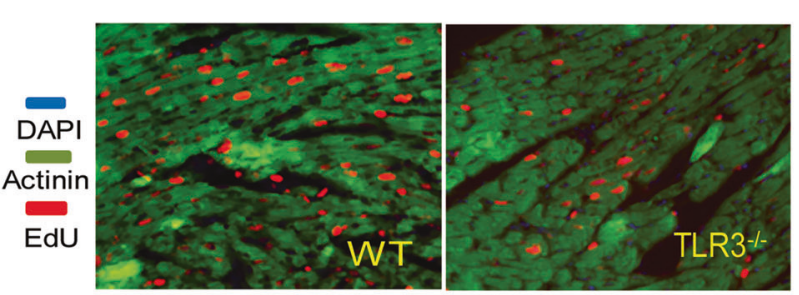

C
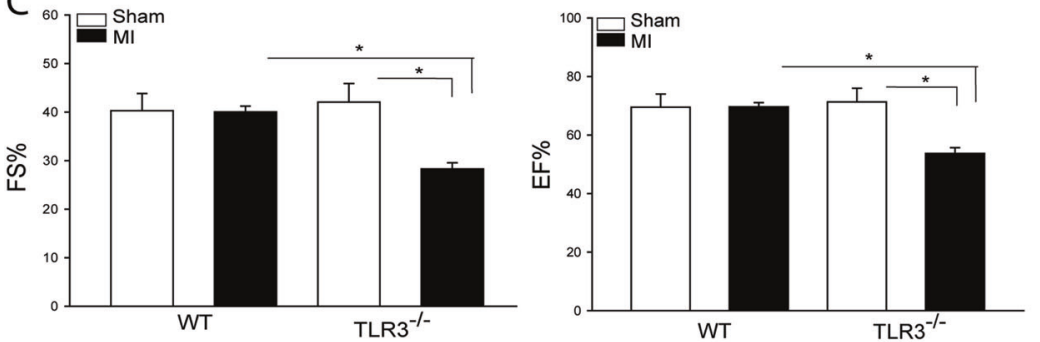
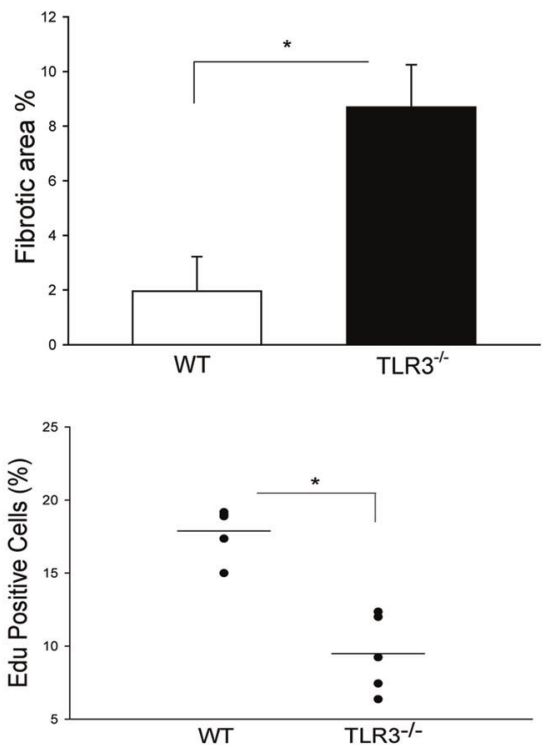

$E$
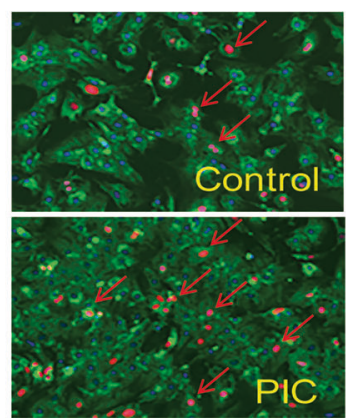

$\mathrm{F}$

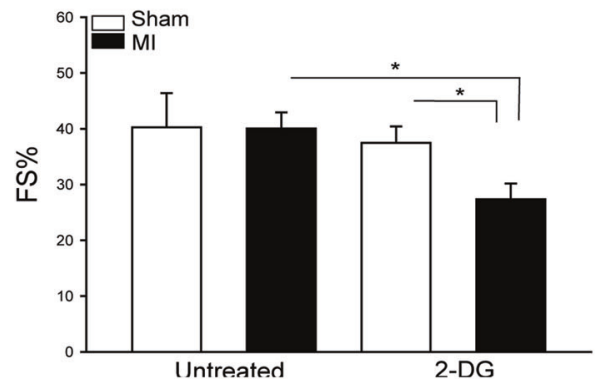

vector control. Fig. 3b, c show that activated YAP1 transfection significantly promotes neonatal cardiomyocyte proliferation. Inhibition of YAP1 with specific siRNA for

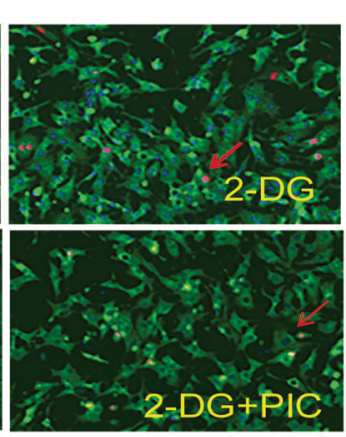

D

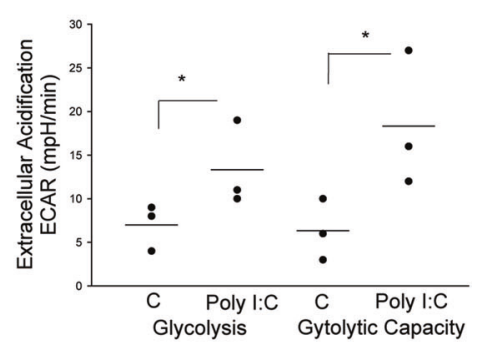

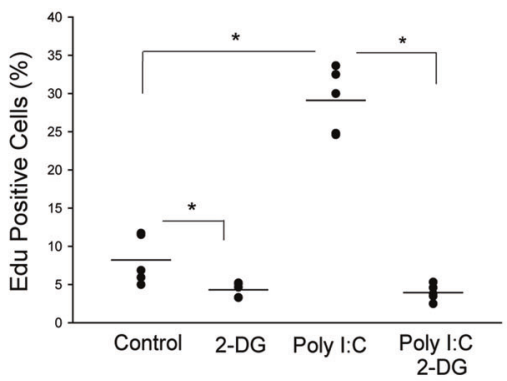

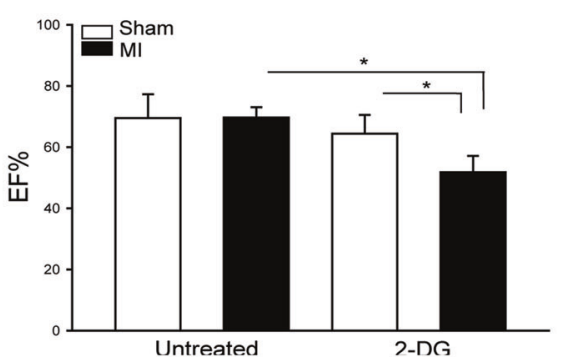

YAP1 (Fig. 3e) or YAP1 inhibitor, verteporfin (Fig. 3f) markedly suppresses Poly (I:C)-induced YAP1 expression, YAP1 nuclear translocation (Fig. 3g) and proliferation of 
Fig. 1 TLR3 deficiency impaired regeneration and repair of damaged hearts following myocardial infarction1 day old (P1) neonatal wild type (WT) and TLR3 deficient $\left(\mathrm{TLR}^{-/-}\right.$) mice were subjected to myocardial infarction (MI). Hearts were harvested 21 days after MI. a Heart tissue sections were stained with hematoxylin and eosin (H\&E) or Masson-trichrome dye after MI. Fibrous area was quantified by image analysis in Masson-trichrome staining. b TLR3 deficiency reduced neonatal cardiomyocyte proliferation. EdU incorporation into the nuclei of cardiomyocytes from $\mathrm{TLR}^{-1-}$ neonatal mice was significantly reduced after MI. c TLR3 ${ }^{-/-}$impaired cardiac functional recovery. 1 day old (P1) neonatal wild type (WT) and TLR3 deficient mice were subjected to myocardial infarction (MI) and cardiac function was measured by echocardiography. $\mathbf{d}$ The TLR3 ligand, Poly (I: C) increased glycolysis and glycolytic capacity in isolated neonatal cardiomyocytes. e Inhibition of glycolysis by 2-Deoxy-D-glucose (2DG) attenuated Poly (I:C) induced increases in EdU incorporation into the nuclei of neonatal cardiomyocytes $\mathbf{e}$ and cardiac functional recovery of P1 WT neonatal mice after MI f. $n=4-8$ /group. * $p<0.05$ compared with indicated groups

neonatal cardiomyocytes (Fig. 3d). The data show that activation of YAP1 is necessary for Poly (I:C)-induced proliferation of neonatal cardiomyocytes.

\section{TLR3 Ligand, Poly (I:C) Suppresses YAP1 Phosphorylation, Leading to YAP1 Activation}

To investigate whether the TLR3 ligand, Poly (I:C) induces YAP1 expression at transcriptional or post-transcriptional levels, we analyzed mRNA levels of YAP/TAZ in neonatal cardiomyocytes treated with Poly (I:C). Figure 4a shows that Poly (I:C) treatment did not alter the levels of YAP/ TAZ mRNAs, indicating that TLR3-medicated YAP1 activation is through post-translational modification.

YAP1 contains two main sites for the phosphorylation at S127 and S397 [16]. Phosphorylation of S127 promotes YAP1 binding with protein 14-3-3, thus preventing its translocation into the nucleus [16]. Phosphorylation of S397 facilitates YAP1 degradation [16, 23]. Therefore, dephosphorylation of YAP1 will lead to activation and nuclear translocation. As shown in Fig. 4b, Poly (I:C) treatment significantly decreases the levels of phosphorylated YAP1 at both S127 and S397 in a time dependent manner, and markedly increases total YAP1 levels. However, Poly (I:C) stimulation did not alter the levels of phosphorylated and total YAP1 in cardiomyocytes isolated from TLR $3^{--}$neonatal mice (Supplemental Figure 1A). The data suggest that Poly (I:C) induces YAP1 activation by suppressing YAP1 phosphorylation.

\section{Suppression of YAP1 Phosphorylation by Poly $(\mathrm{I}: \mathrm{C})$ is Mediated Through Dephosphorylation of LATS1 and MOB1}

LATS1/2 phosphorylates YAP1 at both the S127 and S397 sites, resulting in inactivation or degradation of YAP1 $[16,23]$. MOB1 is an adaptor protein of Hippo signaling and interacts with LATS1/2 to promote YAP1 phosphorylation [24]. In contrast, decreased LATS1 and MOB1 phosphorylation will release their inhibitory effect on YAP/TAZ activation $[16,24]$. We examined the effect of Poly (I:C) treatment on LATS1 and MOB1 phosphorylation in neonatal cardiomyocytes. Fig. 4c shows that Poly (I:C) treatment markedly reduced the levels of LATS1 and MOB1 (Fig. 4b) phosphorylation in a time dependent manner. However, Poly (I:C) treatment did not alter the levels of phosphorylated LATS1 and MOB1 in cardiomyocytes isolated from $\mathrm{TLR}^{-/-}$neonatal mice (Supplemental Fig. 1B and C). The data suggest that Poly (I:C) induced YAP1 activation is mediated through induction of LATS1 and MOB1 dephosphorylation.

\section{TLR3 Ligand, Poly (I:C) Induces an Interaction Between PP1a with LATS1 and MOB1}

Protein phosphorylase 1 (PP1) plays an important role in the induction of protein dephosphorylation [25, 26]. We investigated whether Poly (I:C) could induce an interaction between PP1a and LATS1, resulting in LATS1 and the downstream effector YAP1 dephosphorylation in the neonatal cardiomyocytes. As shown in Fig. 5a, Poly (I:C) treatment significantly promotes the interaction between PP1a and LATS1 as evidenced by showing high levels of LATS1 in the PP1a immunoprecipitates. Poly (I:C) treatment also markedly promotes the interaction of PP1a with MOB1 (Fig. 5a). In contrast, Poly (I:C) stimulation did not induce an interaction between PP1a and LATS1 or MOB1 in cardiomyocytes isolated from TLR $3^{--}$neonatal mice (Supplemental Fig. 1D). The data indicates that PP1a is involved in Poly (I:C) induced decreases in the levels of LATS1 and MOB1 phosphorylation.

To confirm our observation, we treated neonatal cardiomyocytes with a PP1a inhibitor, Okadaic acid and observed that PP1a inhibition abolished Poly (I:C) induced decreases in the levels of phosphorylated LATS1 and MOB1 (Fig. 5b). PP1a inhibition also increased YAP1 phosphorylation, resulting in decreases in the levels of YAP1 (Fig. 5b). In addition, PP1a inhibition markedly attenuated Poly (I:C) induced YAP1 nuclear translocation (Fig. 5c) and neonatal cardiomyocyte proliferation (Fig. 5d). The data suggest that Poly (I:C) induced YAP1 activation and nuclear translocation are mediated by promoting the interaction of PP1a with LATS1 and MOB1, resulting in inactivation of both LATS1 and MOB1 through their dephosphorylation.

\section{TLR3 Ligand, Poly (I:C) Induces Glycolysis Mediated PP1a Dependent LATS1 and YAP1 Dephosphorylation}

To investigate whether glycolysis plays a role in Poly (I:C) induced the interaction of PP1a with LATS1 and decreased levels of phosphorylated LATS1 and YAP1, we treated 
A
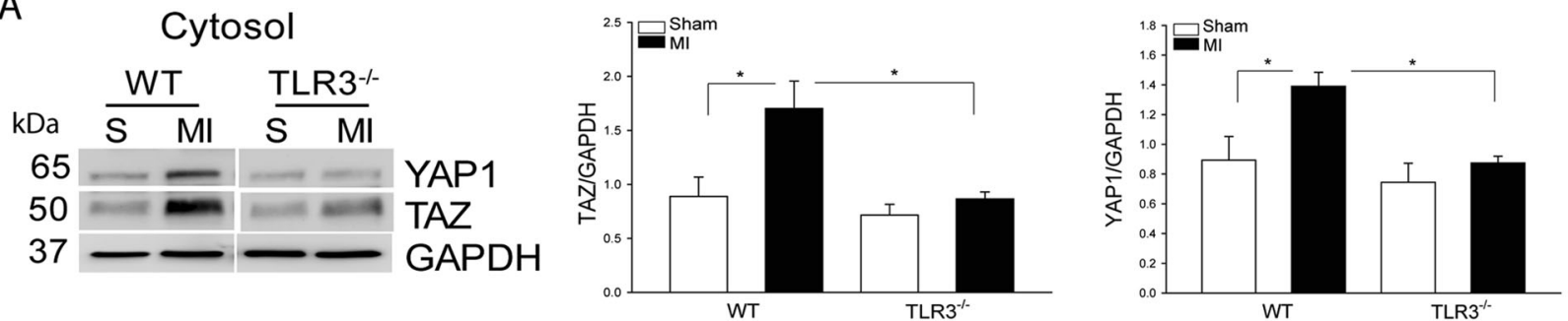

B
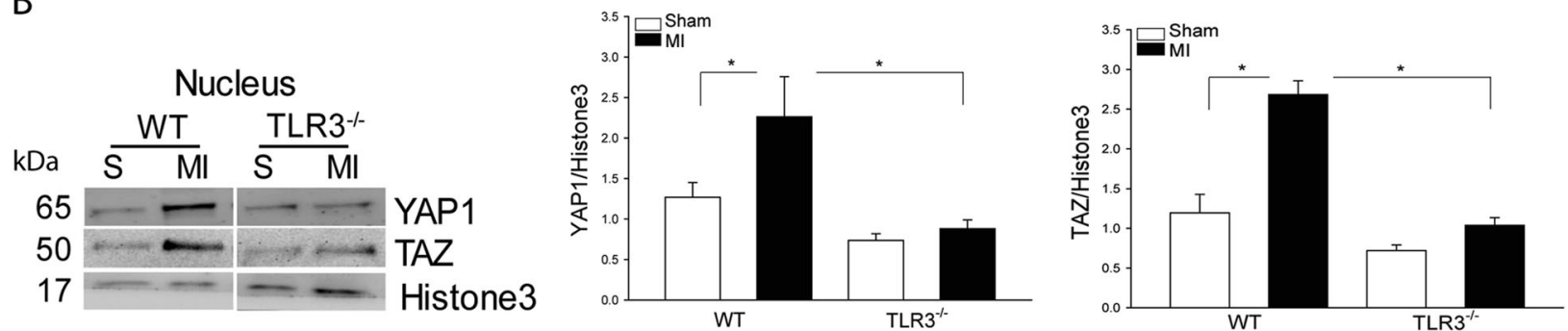

C
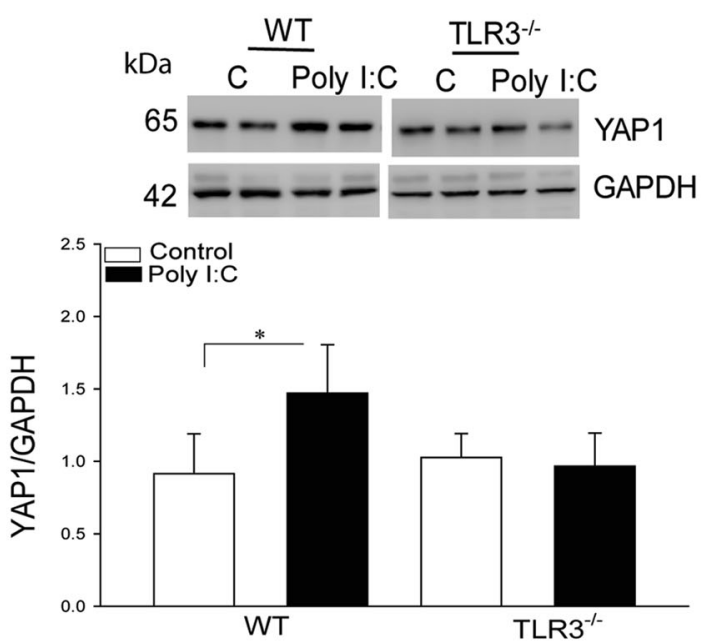

$E$

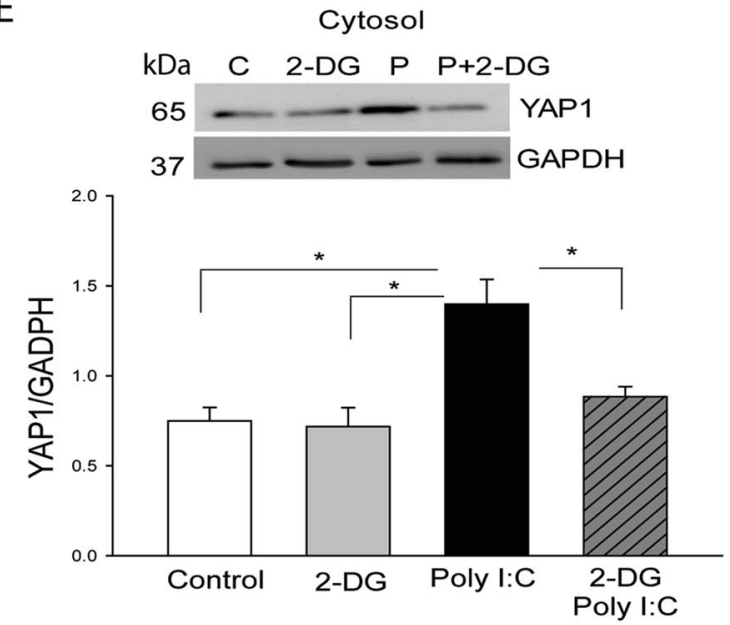

Fig. 2 Myocardial infarction induced increases in YAP1/TAZ expression and nuclear translocation in WT neonatal hearts, but not in TLR3 deficient neonatal mice. 1 day old (P1) neonatal wild type (WT) and TLR3 deficient $\left(\right.$ TLR $^{-1-}$ ) mice were subjected to (MI). a, b Cytosolic and nuclear proteins were isolated from the neonatal hearts for analysis of YAP and TAZ levels in the cytosol $\mathbf{a}$ and the nuclei $\mathbf{b}$.
D
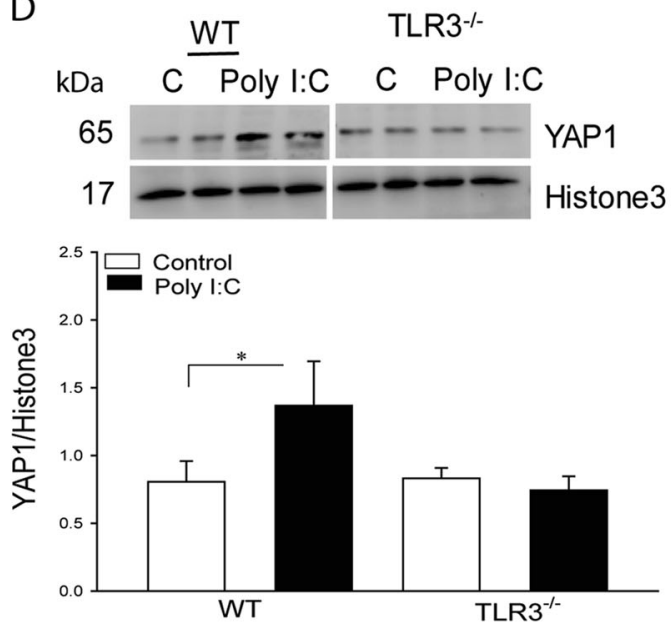

$\mathrm{F}$
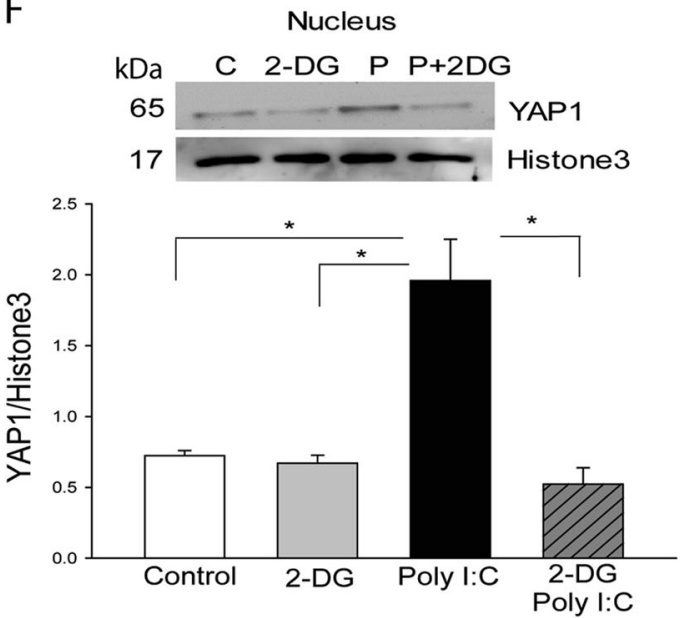

c, d The TLR3 ligand, Poly I:C increased the level of YAP1 in the cytosol $\mathbf{c}$ and promotes YAP1 nuclear translocation $\mathbf{d}$ in WT neonatal cardiomyocytes, but not in $\mathrm{TLR}^{-/-}$neonatal cardiomyocytes. e, f Inhibition of glycolysis by 2-DG prevents Poly I:C-induced increases in YAP1 expression in cytosol e and nuclear translocation f. $n=3-6 /$ group. $* p<0.05$ compared with indicated groups 
A

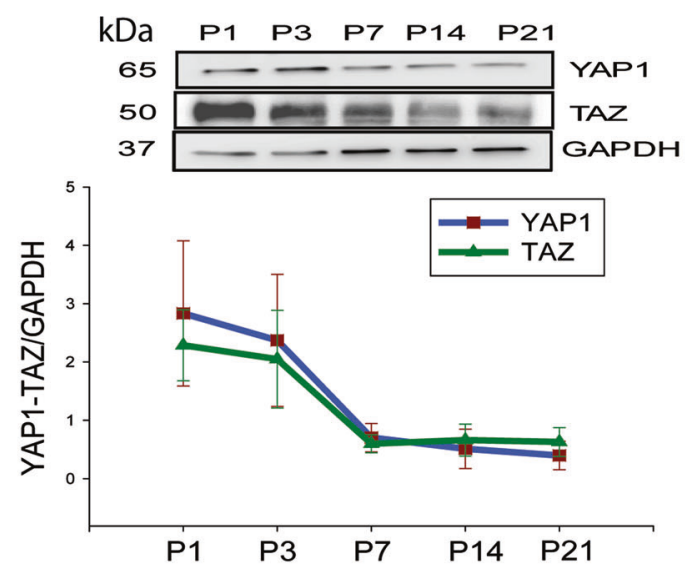

C

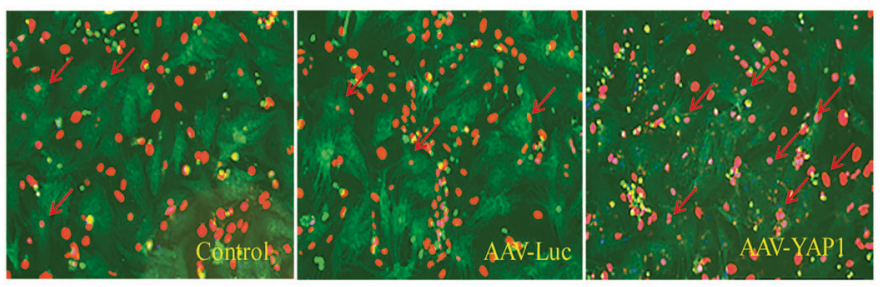

D

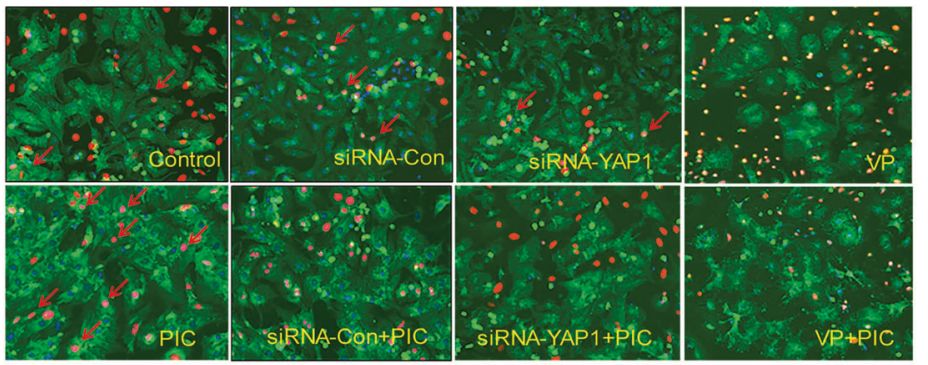

B $\quad \mathrm{kDa}$

65

37
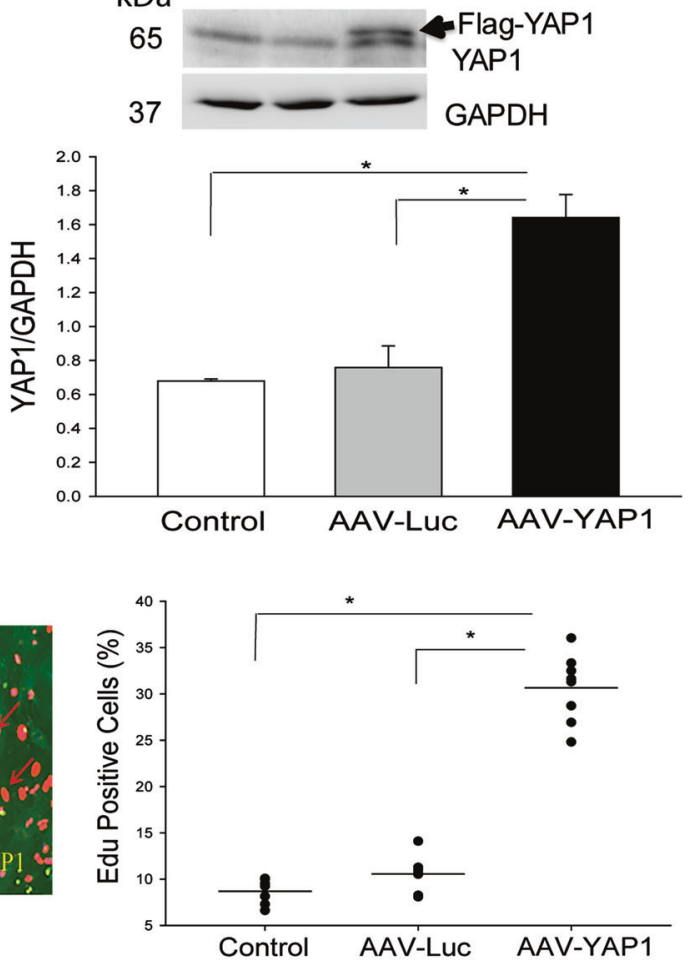

E
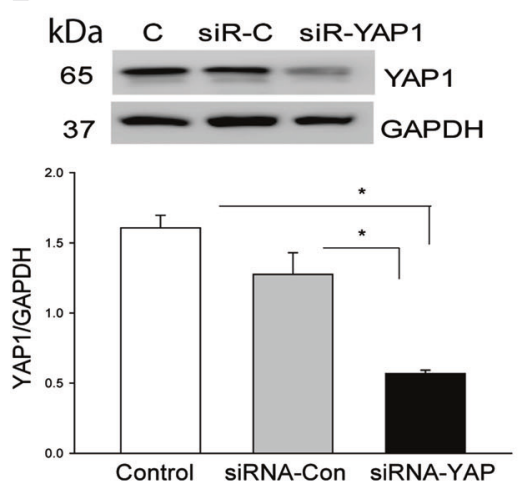

$\mathrm{F}$
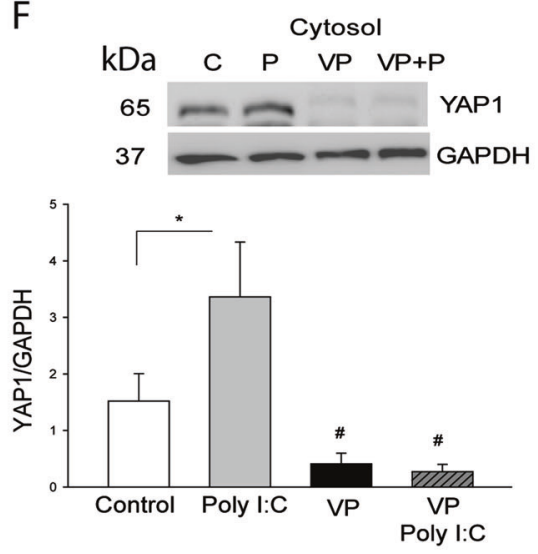

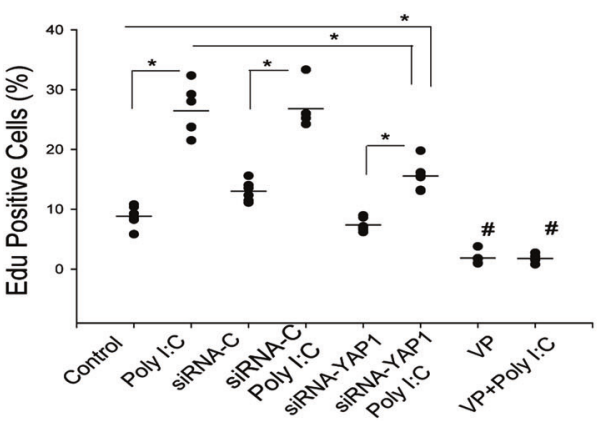

G
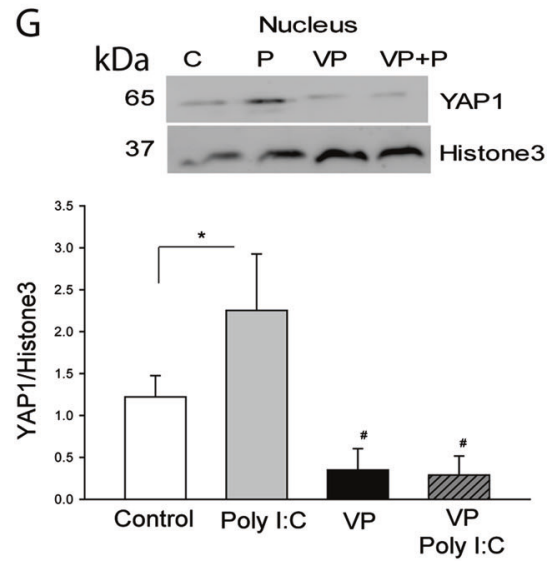

Fig. 3 YAP1 activation is required for TLR3-mediated neonatal cardiomyocyte proliferation. a The levels of YAP/TAZ in the myocardium are greater in $\mathrm{P} 1$ and $\mathrm{P} 3$ neonatal mice and are gradually decreased in P7, P14 and P21 neonatal mice. b, c Increased YAP levels in neonatal cardiomyocytes by transfection of AAV virus expressing activated YAP1 increased YAP1 levels (B) and promoted neonatal cardiomyocyte proliferation c. d-f Inhibition of YAP1 expression by specific siRNA for YAP1 or YAP inhibitor, verteporfin (VP) markedly suppressed Poly I:C (PIC)-induced neonatal cardiomyocyte proliferation d, YAP1 expression in cytosol $\mathbf{e}, \mathbf{f}$ and nuclear translocation g. $n=3-8 /$ group. $* p<0.05$ compared with indicated groups. ${ }^{\#} p<0.05$ compared with the control group 
A

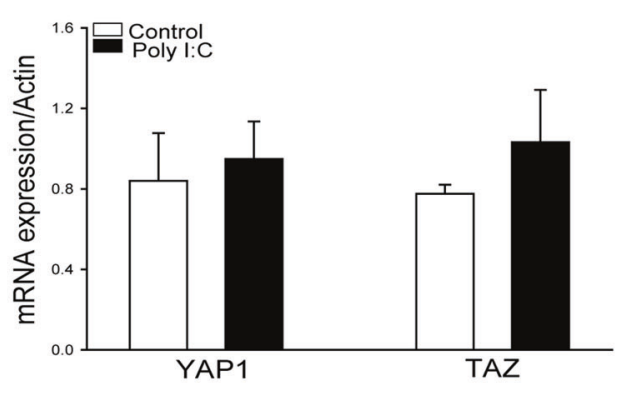

B
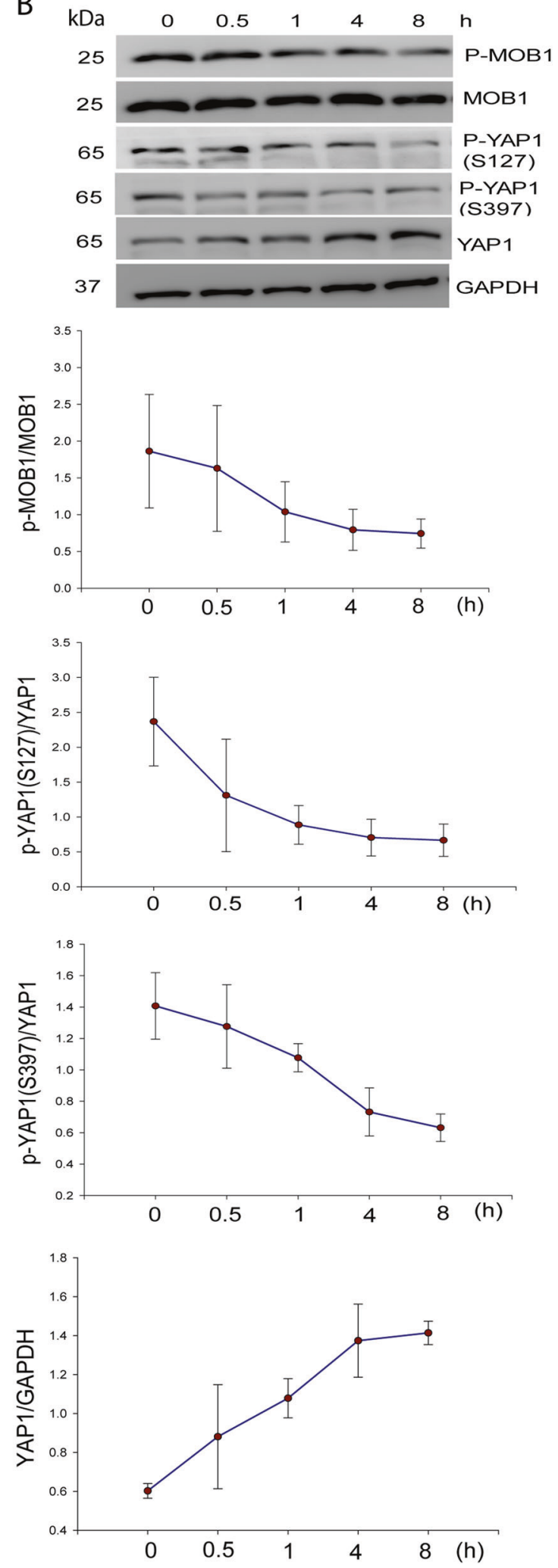

and Western blot analysis of LATS, MOB1 and YAP. a Poly (I:C) treatment had no effect on YAP1 or TAZ mRNA expression. b, $\mathbf{c}$ Poly (I:C) treatment significantly reduced the phosphorylation of LATS1 b, MOB1 and YAP1 c. $n=3-5 /$ group
Fig. 4 The TLR3 ligand, Poly (I:C) reduced LATS1, MOB1, and YAP1 phosphorylation in neonatal cardiomyocytesNeonatal cardiomyocytes were treated with Poly (I:C) for different time points. Total RNA and cytosolic proteins were isolated for PCR assay of mRNA
P-LATS1

LATS1

GAPDH ACTIN

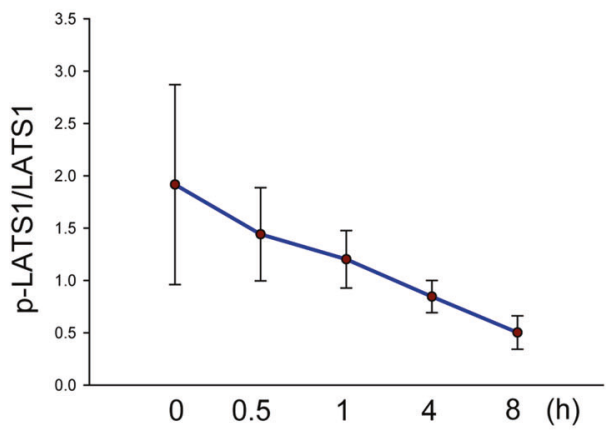


A

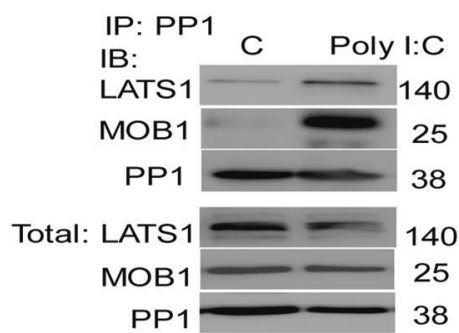

B

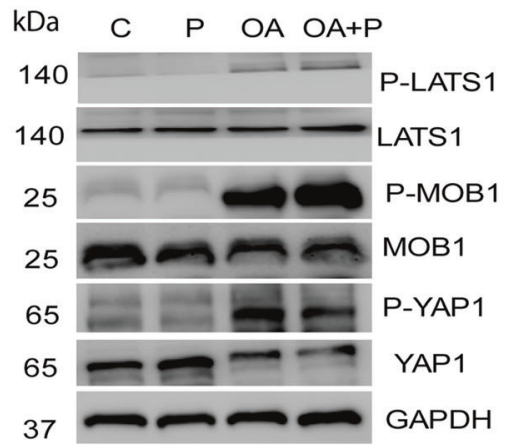

C
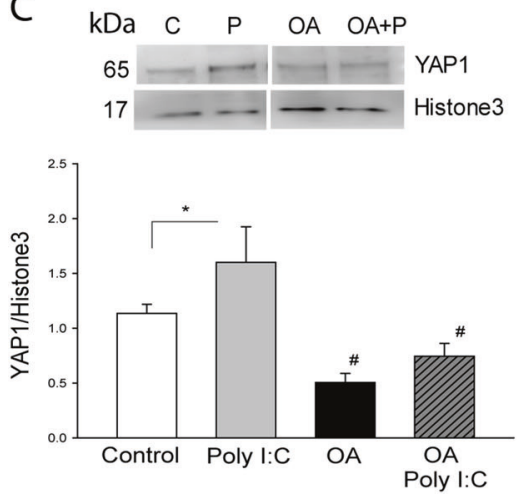

D
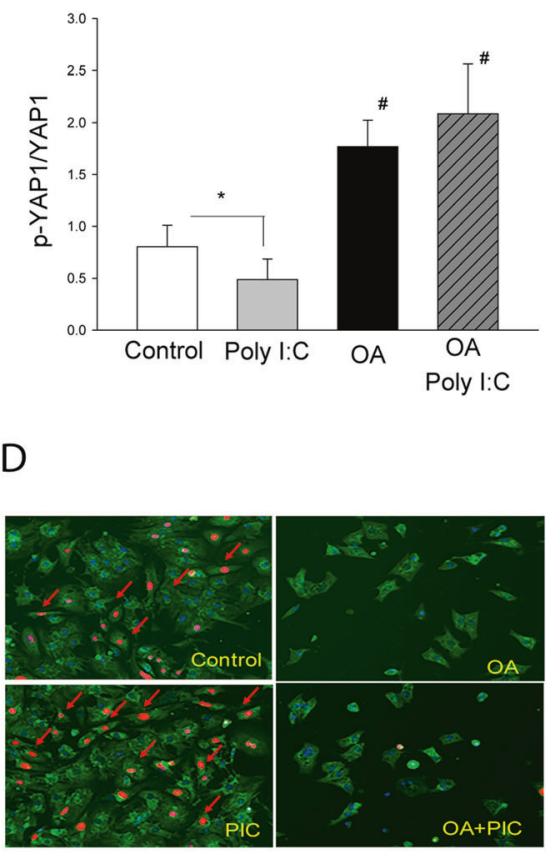
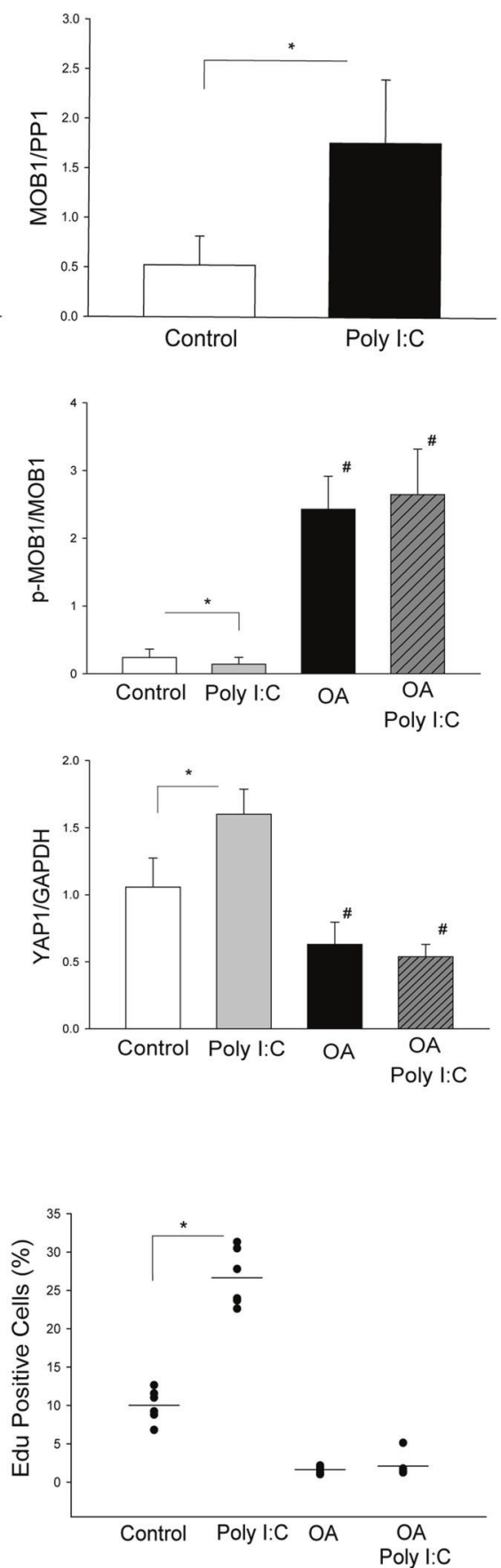

Fig. 5 PP1a is involved in Poly (I:C) induced LATS1 and MOB1 dephosphorylation, YAP1 activation, and neonatal cardiomyocyte proliferationa Poly (I:C) treatment induced an interaction of PP1 with LATS1 and MOB1 in neonatal cardiomyocytes. Neonatal cardiomyocytes were treated with Poly (I:C) and cellular proteins were isolated for the immunoprecipitation with specific anti-PP1 antibody. The immunoprecipitates were subjected to immunoblot with antiLATS1 and anti-MOB1, respectively. b PP1 inhibitor Okadaic acid

neonatal cardiomyocytes with 2-DG in the presence or absence of Poly (I:C) and examined the interaction of PP1a with LATS1 and the levels of LATS1 and YAP1
(OA) treatment increased phosphorylation of LATS, MBO1, and YAP1 and attenuated Poly (I:C) induced decreases in the phosphorylation levels of LATS1, MOB1 and YAP1 in the neonatal cardiomyocytes. c, d PP1 inhibitor OA treatment prevented Poly (I:C) induced YAP1 nuclear translocation $\mathbf{c}$ and neonatal cardiomyocyte proliferation d. $n=3-8$ /group. $* p<0.05$ compared with indicated groups. ${ }^{*} p<0.05$ compared with the control group

phosphorylation. As shown in Fig. 6a, Poly (I:C) treatment significantly strengthened the interaction between PP1a and LATS1. However, inhibition of glycolysis by 2-DG 
A

IP: PP1 $\alpha$

IB:

LATS1

PP1

Total: LATS1

$\mathrm{PP} 1 \alpha$

$$
\text { C P 2-DG 2-DG+P }
$$
$140^{\mathrm{kDa}}$
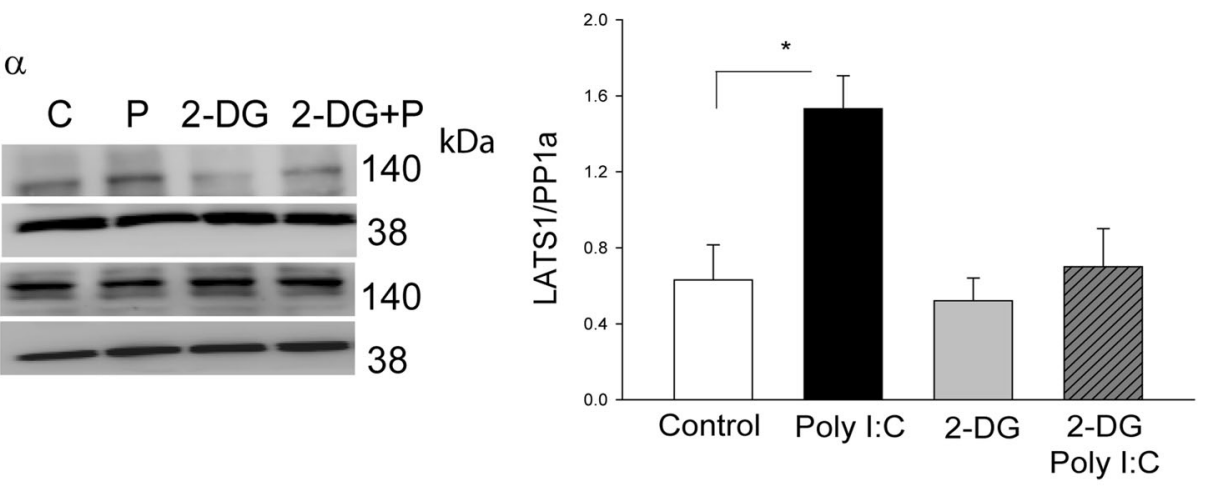

B
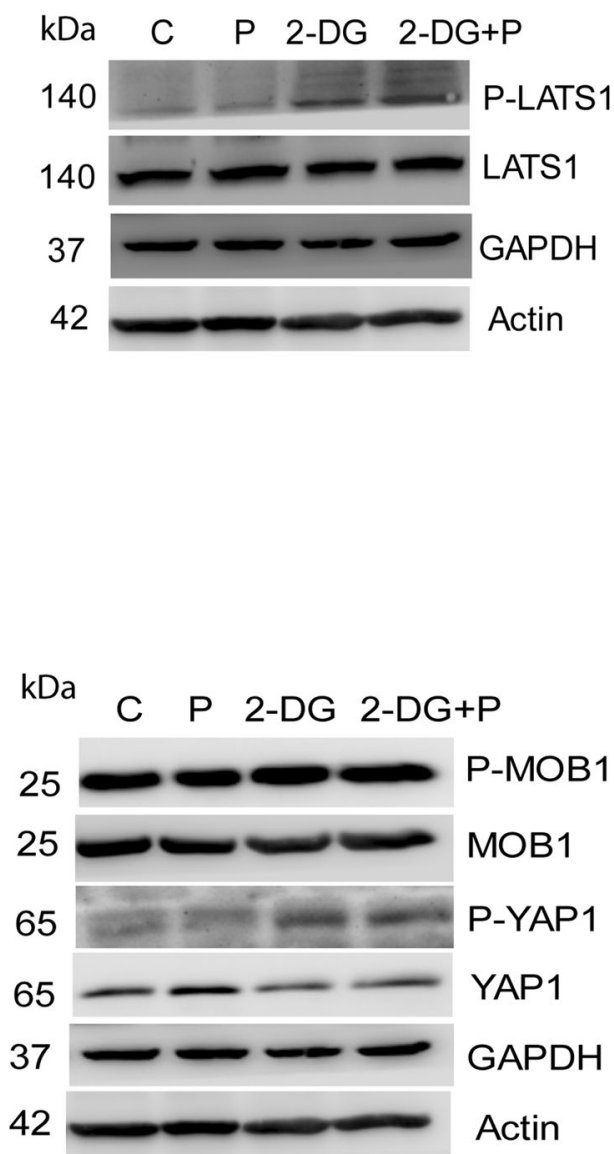

Fig. 6 Glycolysis induced by Poly (I:C) mediates PP1a dependent LATS1 and YAP1 dephosphorylation. a Treatment of neonatal cardiomyocytes with the glycolysis inhibitor, 2-DG, attenuated Poly I:Cinduced interaction of PP1 with LATS1. b, $\mathbf{c}$ Inhibition of glycolysis with 2-DG increased the levels of phosphorylated LATS $\mathbf{b}$ and YAP1
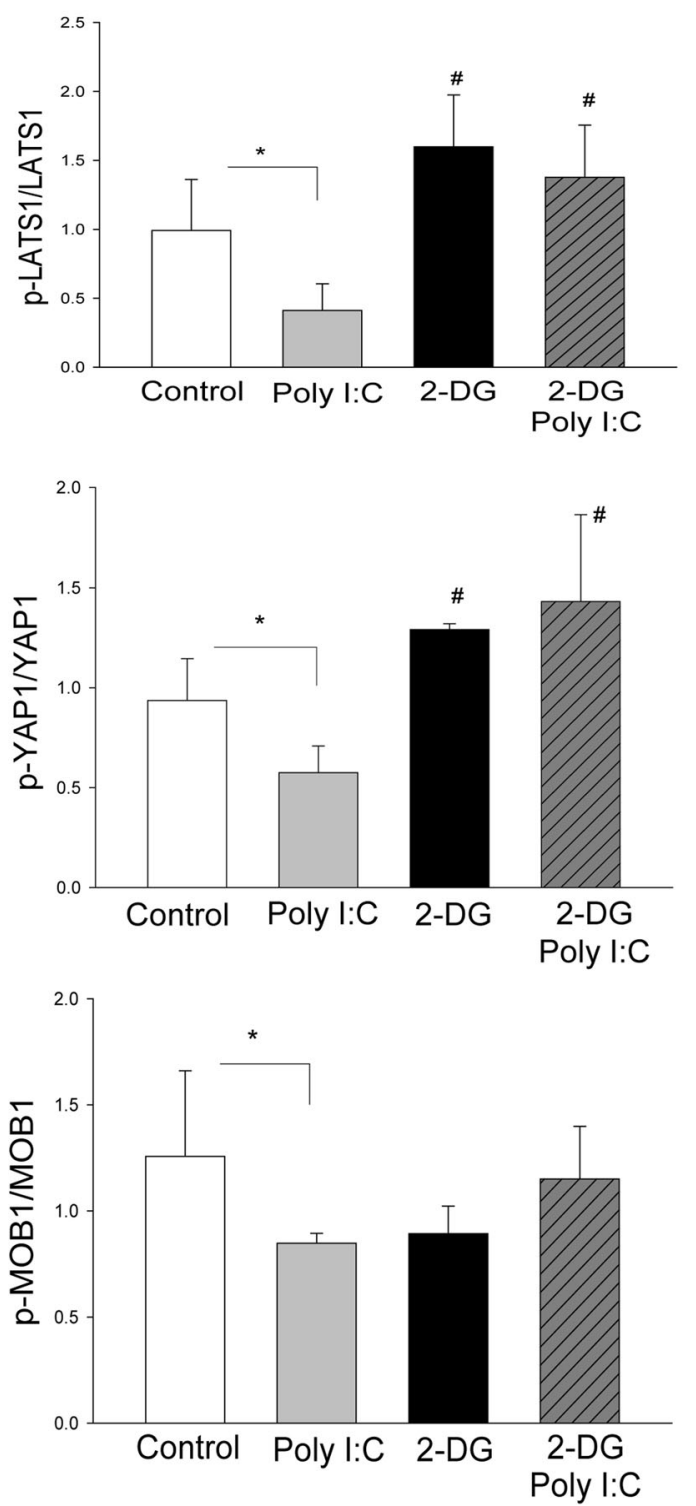

c in neonatal cardiomyocytes. $\mathbf{c} 2-\mathrm{DG}$ treatment did not alter Poly (I:C) decreased MOB1 phosphorylation. $n=3-5$ /group. $* p<0.05$ compared with indicated groups. ${ }^{\#} p<0.05$ compared with the control group 
Fig. 7 AMPK is involved in TLR3 ligand, Poly (I:C)-induced YAP1 activation and neonatal cardiomyocyte proliferation. a Treatment of neonatal cardiomyocytes with Poly (I:C) significantly decreased the levels of phosphorylated AMPK in neonatal cardiomyocytes. b Inhibition of glycolysis by 2-DG increased AMPK

phosphorylation and abolished Poly (I:C) induced decreases in the levels of dephosphorylated AMPK. c, d Treatment of neonatal cardiomyocytes with an AMPK activator, metformin, prevents Poly (I:C) induced dephosphorylation of AMPK $\mathbf{c}$ and YAP1 d. e, $\mathbf{f}$ Treatment of neonatal cardiomyocytes with the AMPK activator metformin prevented Poly (I:C) induced YAP1 nuclear translocation e and neonatal cardiomyocyte proliferation f. $n=3-6 /$ group. $* p<0.05$ compared with indicated groups. ${ }^{*} p<0.05$ compared with the control group
A

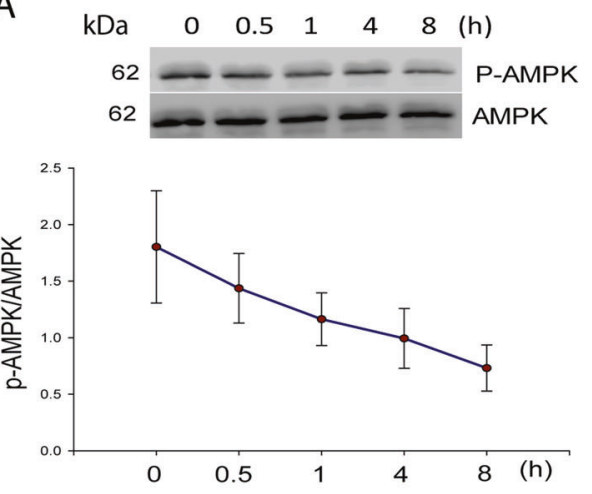

C
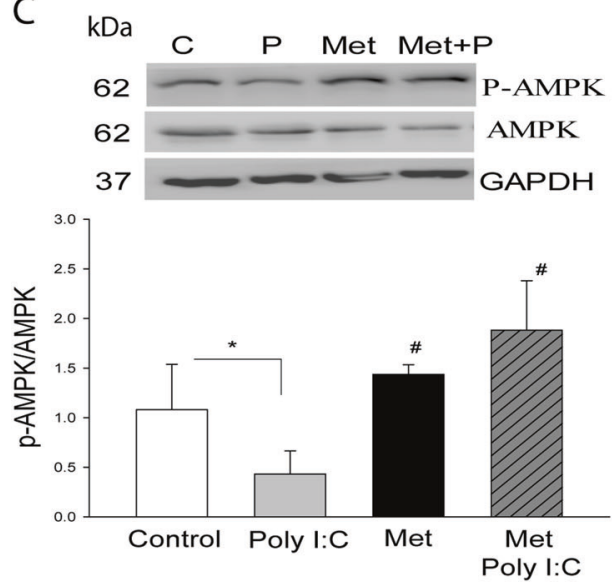

E

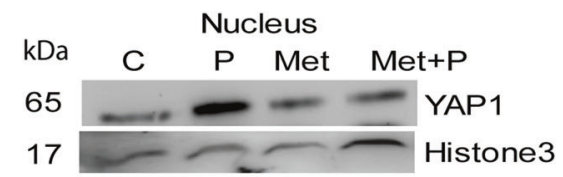

F

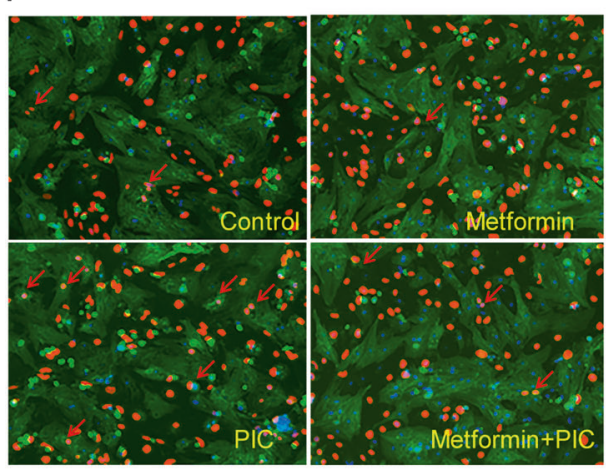

B
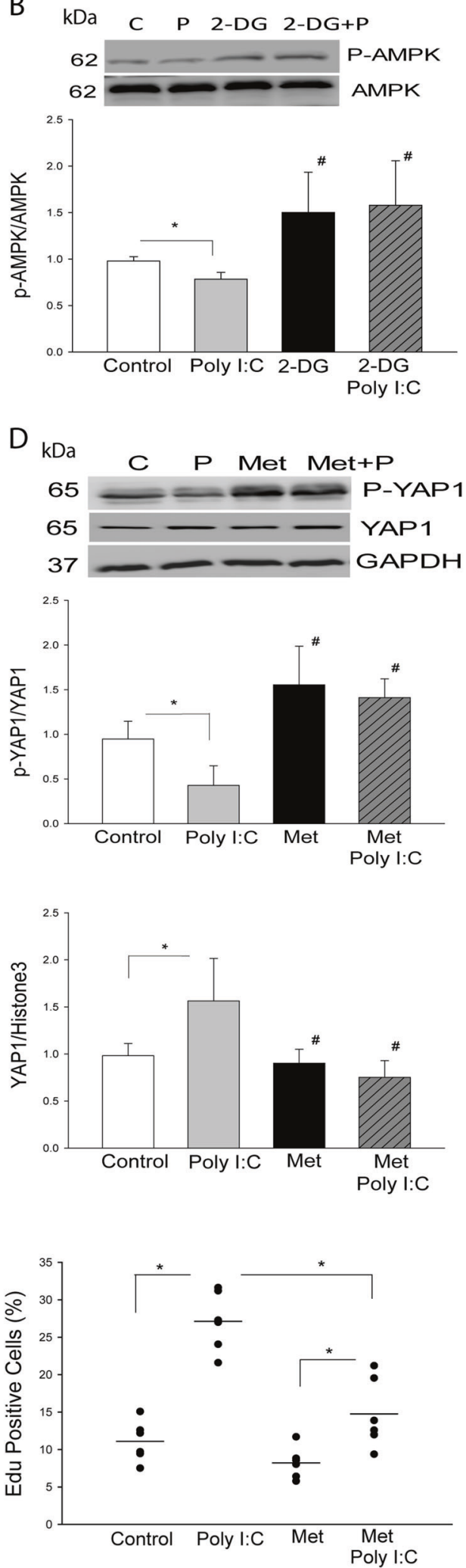

prevented Poly I:C-induced interaction of PP1a with LATS1, suggesting that Poly I:C-induced interaction of PP1a with LATS1 is mediated through glycolytic metabolism. To further confirm the role of glycolysis in Poly I:C-induced dephosphorylatoin of LATS1 and YAP1, we treated neonatal cardiomyocytes with 2-DG and 

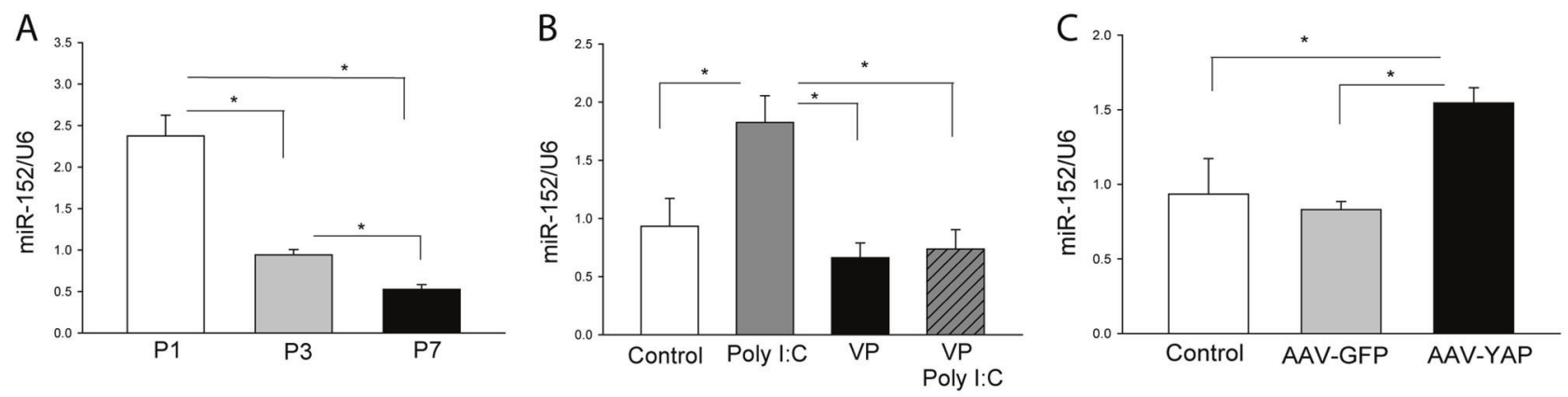

D
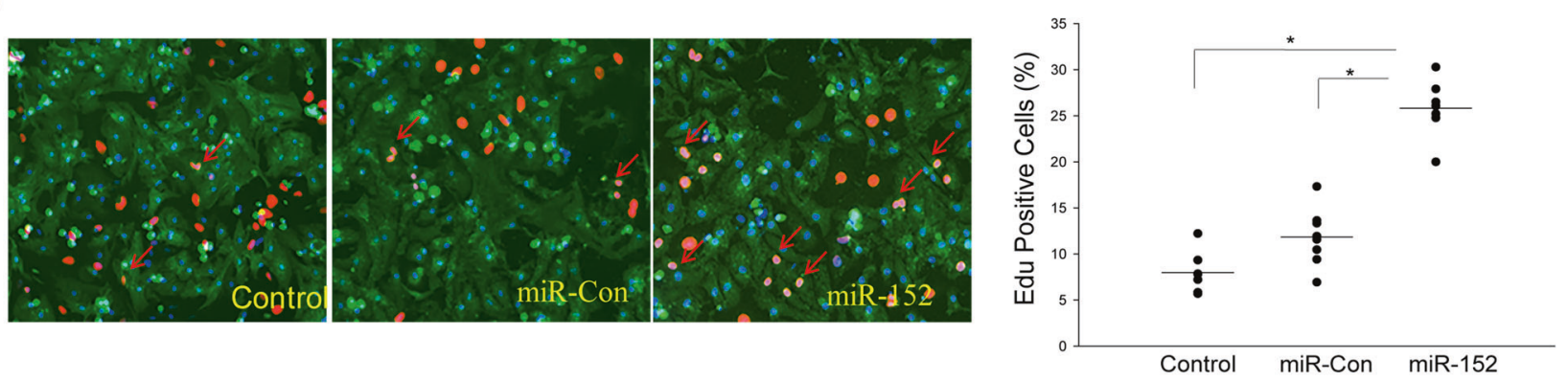

E
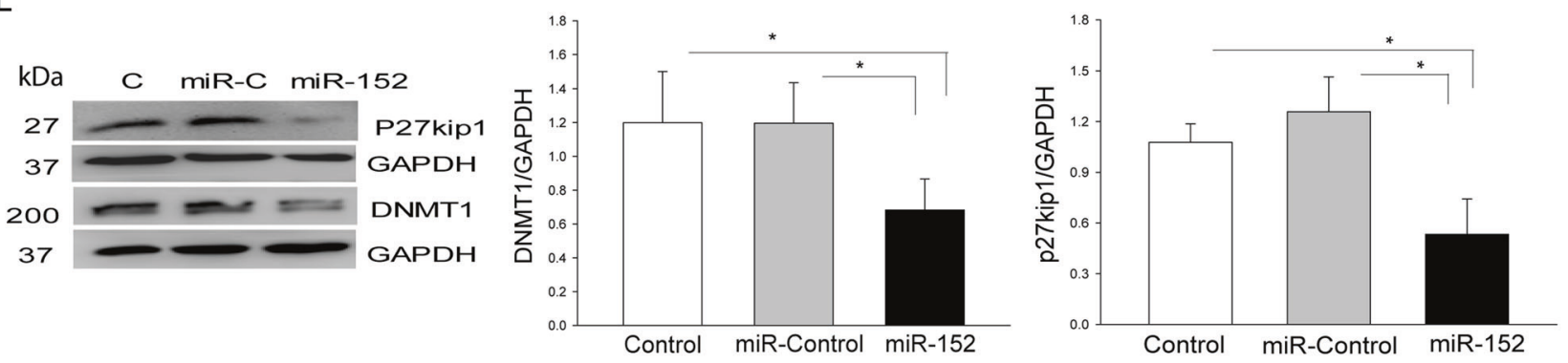

$\mathrm{F}$
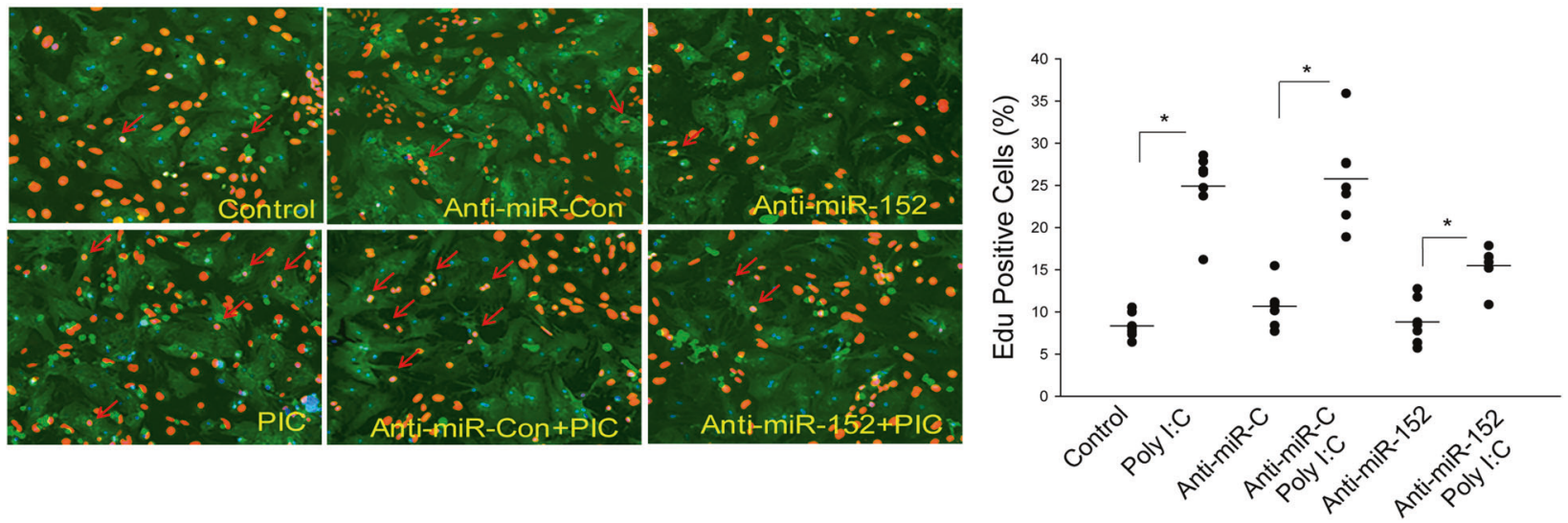

Fig. 8 MicroRNA-152 (miR-152) is involved in TLR3-mediated YAP1 activation and neonatal cardiomyocyte proliferation. a The levels of miR-152 expression are greater in the myocardium of P1 neonatal mice and gradually decrease in $\mathrm{P} 3$ and $\mathrm{P} 7$ neonatal hearts. b, c YAP1 is required for miR-152 expression in neonatal cardiomyocytes. b Treatment of neonatal cardiomyocytes with YAP inhibitor, VP abolished Poly (I:C) induced increases in miR-152 expression. c Transfection of neonatal cardiomyocytes with AAV virus carrying activated YAP1 increased miR-152 expression. d, e Transfection of neonatal cardiomyocytes with miR-152 mimics promotes neonatal cardiomyocyte proliferation $\mathbf{d}$ and represses p27kip1 and DNMT1 expression e. f Treatment of neonatal cardiomyocytes with anti-miR152 mimics attenuates Poly (I:C) induced neonatal cardiomyocyte proliferation. $n=3-6$ /group. $* p<0.05$ compared with indicated groups. ${ }^{\#} p<0.05$ compared with the control group 
examined the levels of LATS1, MOB1 and YAP1 phosphorylation. As shown in Fig. 6b, c, 2-DG treatment alone markedly increased the levels of LATS1 and YAP1 phosphorylation. Importantly, 2-DG administration also abolished Poly (I:C) suppressed phosphorylation of LATS1, thereby increasing YAP1 phosphorylation. The data suggest that glycolysis is involved in Poly (I:C) induced decreases in the levels of LATS1 phosphorylation by promoting the interaction between PP1a and LATS1, leading to YAP1 activation. We observed that 2-DG treatment did not alter Poly (I:C) induced decreases in the levels of MOB1 phosphorylation (Fig. 6c), indicating that in addition to glycolysis, other mechanisms may be involved in Poly (I:C) induced MOB1 dephosphorylation.

\section{TLR3 Ligand, Poly (I:C) Modulates AMPK Phosphorylation and YAP1 Activation via a Glycolytic-Dependent Mechanism}

AMPK is abundantly expressed in the heart and plays an important role in the regulation of cellular metabolism [27]. AMPK activity is regulated by glycolysis and involves energy stress-induced inactivation of YAP1 [28]. We examined whether Poly (I:C) would regulate AMPK activation via glycolysis. As shown in Fig. 7a, following Poly (I:C) treatment, the levels of phosphorylated AMPK are gradually reduced in a time dependent manner. Administration of 2-DG abolished Poly (I:C)-suppressed AMPK phosphorylation and significantly increase the levels of phosphorylated AMPK (Fig. 7b). However, Poly (I:C) treatment did not alter the levels of phosphorylated AMPK in neonatal cardiomyocytes isolated from $\mathrm{TLR}^{-/-}$mice (Supplemental Fig. 1E). The data indicate that Poly (I:C) decreases AMPK phosphorylation via glycolysis.

To examine whether AMPK would play a role in the regulation of YAP1 activation, we treated neonatal cardiomyocytes with an AMPK specific activator, metformin and examined YAP1 activation as well as the proliferation of neonatal cardiomyocytes in the presence and absence of Poly (I:C). As shown in Fig. 7c, treatment of the cells with metformin abolished Poly (I:C) induced decreases in AMPK phosphorylation. Importantly, metformin treatment also abolished Poly (I:C) induced dephosphorylation of YAP1 (Fig. 7d), YAP1 nuclear translocation (Fig. 7e), and neonatal cardiomyocyte proliferation (Fig. 7f). The data suggest that activation of AMPK negatively regulates YAP1 activation and nuclear translocation as well as neonatal cardiomyocyte proliferation.

\section{Activation of YAP1 Regulates miR-152 Expression in Neonatal Cardiomyocytes}

To investigate the mechanisms by which activated YAP1 induces neonatal cardiomyocyte proliferation, we examined the role of microRNA-152 (miR-152) in TLR3-mediated YAP1 dependent neonatal cardiomyocyte proliferation. It is well known that miR-152 targets cell cycle entry proteins p27kip1 and DNA methyltransferase1 (DNMT1) which are important proteins in the regulation of cell proliferation [29, 30]. As shown in Fig. 8a, miR-152 levels are the highest in P1 neonatal hearts but are gradually reduced in P3 and P7 neonatal hearts. In vitro treatment of $\mathrm{TLR} 3^{-/}$neonatal cardiomyocytes with Poly (I:C) did not alter the levels of miR-152 (Supplemental Fig. 2A), but significantly increased expression of miR-152 in WT neonatal cardiomyocytes (Fig. 8b). However, inhibition of YAP1 with a YAP1 inhibitor (VP) prevented Poly (I:C) induced increases in miR-152 expression (Fig. 8b). In contrast, increased YAP1 activation by transfection of neonatal cardiomyocytes with AAV-YAP1 markedly increases the levels of miR-152 expression (Fig. 8c). The data suggest that activation of YAP1 regulates the expression of miR-152 in neonatal cardiomyocytes.

\section{MiR-152 Contributes to TLR3-Mediated Cardiomyocyte Proliferation}

To investigate the role of miR-152 in TLR3-mediated neonatal cardiomyocyte proliferation, we transfected neonatal cardiomyocytes with miR-152 mimics or anti-miR152 mimics, respectively (Supplemental Fig. 2B) and examined the proliferation of neonatal cardiomyocytes. As shown in Fig. 8d, transfection of miR-152 mimics significantly promotes the proliferation of neonatal cardiomyocytes as evidenced by incorporation of EdU into the neonatal cardiomyocytes. MiR-152 mimic transfection markedly suppresses the expression of p27kip and DNMT1 in the neonatal cardiomyocytes (Fig. 8e). P27kip1 is a cyclindependent kinase inhibitor while DNMT1 plays a critical role in regulation of the cell cycle [31, 32]. Suppression of p27kip1 and DNMT1 will promote cell cycle entry, leading to proliferation. However, suppression of miR-152 expression by transfection of cells with anti-miR-152 significantly attenuates Poly (I:C) induced cell proliferation (Fig. 8f). The data suggest that an increased level of miR-152 promotes neonatal cardiomyocyte proliferation via suppression of p27kip and DNMT1 expression (Fig. 9).

\section{Discussion}

TLRs are conserved pattern-recognition receptors that are involved in the pathophysiology of myocardial ischemia/ reperfusion (I/R) injury [33]. We have previously reported that TLR3 contributes to acute and early I/R-induced adult myocardial injury [33]. Interestingly, TLR3-mediated signaling is involved in cell reprogramming and tissue regeneration [34-36]. indicating that TLR3 may be 


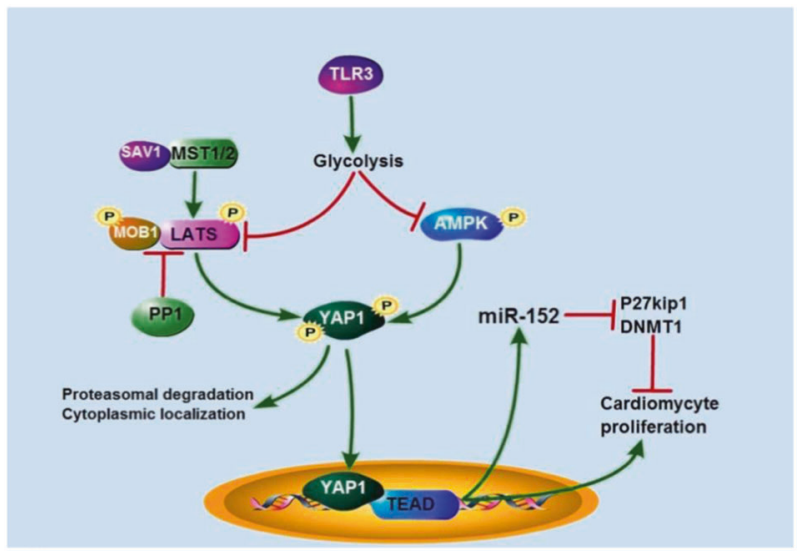

Fig. 9 Illustration of TLR3-mediated YAP1 activation and miR-152 expression in cardiomyocyte proliferationActivation of TLR3 increased glycolysis, resulting in inactivation of LATS and AMPK by reduced phosphorylation. Inactivated LATS and AMPK lead to activation of YAP1 which, as a co-transcriptional factor regulated miR152 expression. miR-152 suppressed p27kip1 and DNMT1 expression, promoting cell proliferation

involved in damaged heart repair and regeneration in the late stage of myocardial ischemic injury. Adult heart has extremely limited capability for regeneration and repair of damaged myocardium [37-39]. Therefore, neonatal heart is an optional model for investigating the role of TLR3 in damaged heart repair and regeneration. The contribution of macrophages to neonatal heart regeneration has been well demonstrated [40]. Therefore, the present study focused on the role of TLR3 in cardiac myocyte proliferation in damaged heart repair and regeneration. TLR3 deficiency impairs neonatal heart repair and regeneration after MI. At present, we do not understand what endogenous ligands might activate TLR3 for repair and regeneration in the damaged neonatal heart. However, the TLR3 ligand, Poly (I:C) markedly enhanced glycolysis in neonatal cardiomyocytes, demonstrating, for the first time to our knowledge, that glycolysis increased by Poly (I:C) plays a critical role in the proliferation of neonatal cardiomyocytes in vitro and heart repair and regeneration in vivo. It is well known that zebrafish display a distinct ability to regenerate their heart following injury. However, another teleost, such as medaka does not have this ability. Lai et al. [41] recently observed delayed and reduced macrophage recruitment in medaka, along with delayed neutrophil clearance. Interestingly, treatment of medaka with the TLR3 ligand, Poly (I:C) significantly enhanced immune cell dynamics and promoted neovascularization, neutrophil clearance, cardiomyocyte proliferation and scar resolution [41]. The data suggest that in addition to increases in glycolysis, Poly (I:C) administration could significantly regulate immune function to promote repair of the damaged heart.
YAP1 activation plays an important role in mediating cell proliferation and differentiation [16, 17]. We observed that the P1 and P3 neonatal hearts have greater levels of YAP1 than that in P7, P14 and P21 neonatal mice, indicating that higher levels of YAP1 in the neonatal heart may be associated with the regenerative capacity within the first week after birth. Interestingly, Bassat et al has recently reported that changes in the composition of the extracellular matrix $(E C M)$ during the first week after birth can affect cardiomyocyte growth and differentiation in mice [42]. These authors have identified a component of neonatal ECM called agrin, which is required for the full regenerative capacity of neonatal mouse hearts. A single in vivo administration of agrin promotes cardiac regeneration in adult mice after myocardial infarction. The mechanisms involve the disassembly of the dystrophin-glycoprotein complex (DGC), and YAP- and ERK-mediated signaling [42]. DGC is a multicomponent transmembrane complex that links the actinin cytoskeleton to ECM. Morikawa et al have found that the DGC component dystroglycan 1 (Dag1) directly binds to the Hippo pathway effector YAP to inhibit cardiomyocyte proliferation in mice [43]. These authors revealed that DGC sequesters pYAP as a mechanism to regulate cardiomyocyte proliferation in postnatal and adult heart [43]. We observed that neonatal hearts have high levels of YAP1 that are associated with aerobic glycolysis in the neonatal hearts. Suppression of glycolysis by 2-DG in neonatal cardiomyocytes prevented Poly (I:C) induced increases in YAP1 expression, suggesting that Poly (I:C) increases glycolysis that regulates YAP1 expression in neonatal cardiomyocytes.

We observed that Poly (I:C) does not alter YAP/TAZ mRNA levels in neonatal cardiomyocytes, indicating that Poly (I:C) may regulate YAP1 activation at the posttranscriptional level. Increased phosphorylation of LATS1 and its adaptor protein MOB1 will result in YAP1 phosphorylation and degradation [16, 24]. We observed that Poly (I:C) treatment significantly decreased the levels of LATS1 and MOB1 phosphorylation. Importantly, inhibition of glycolysis by 2-DG abolished Poly (I:C) induced dephosphorylation of LATS1 but not MOB1. These data suggest that Poly (I:C) induced decreases in the levels of phosphorylated MOB1 may be mediated through a glycolytic independent mechanism. Indeed, we have found that there is an interaction between MOB1 and PP1 following Poly (I:C) treatment of neonatal cardiomyocytes. PP1 is a protein phosphatase that has been reported to interact with LATS1, resulting in inactivation of LATS1/2 by dephosphorylation of LATS1 or direct dephosphorylation of YAP1/TAZ, leading to their activation $[25,26,28]$. Our findings suggest that Poly (I:C) induced YAP1 activation is mediated, in part by promoting a co-association of PP1a with MOB1 and LATS1, resulting in inactivation of both 
MOB1 and LATS1. Indeed, PP1a inhibition by okadaic acid abolished Poly (I:C) induced neonatal cardiomyocyte proliferation.

AMPK has been reported to regulate glycolytic metabolism [27] and suppress YAP1 activation through phosphorylation of LATS1 [28, 44]. We observed that AMPK is involved in Poly (I:C) induced proliferation of neonatal cardiomyocytes. To the best of our knowledge, this is the first report that TLR3 activation decreases AMPK phosphorylation via a glycolytic-dependent mechanism. In contrast, administration of metformin, an AMPK activator, significantly increased AMPK phosphorylation and abolished Poly (I:C) induced YAP1 activation and nuclear translocation as well as proliferation of neonatal cardiomyocytes. Our findings suggest that, in addition to inactivation of LATS1 leading to activation of YAP1 through glycolysis, Poly (I:C) also induces dephosphorylation of AMPK via glycolysis, resulting in YAP1 activation and nuclear translocation in neonatal cardiomyocyte proliferation.

It is well known that activation of YAP1 promotes cell proliferation and differentiation [16, 17]. However, the mechanisms remain elusive. YAP1 is a co-transcriptional factor which can regulate microRNA biogenesis and expression with its DNA binding partner TEAD [45]. To address this issue, we investigated whether activated YAP1/ TAZ could regulate the expression of proteins, such as P27kip1 and DNMT1 (DNA methyltransferase 1), controlling cell cycle entry [31, 32]. P27kip1 is a cyclindependent kinase inhibitor by binding and inhibiting cyclin/ CDKs [32]. DNA methylation in the promoter region of cell cycle related genes is associated with gene repression and prevents cell proliferation. DNMT1 is gradually upregulated during post-neonatal heart development and inhibition of DNMT1 markedly increases cardiomyocyte proliferation [31]. Therefore, it is possible that targeting both P27kip1 and DNMT1 could promote cell proliferation. We demonstrated that activation of YAP1 by Poly (I:C) significantly increases the expression of miR-152 which represses the expression of P27kip1 and DNMT1, resulting in proliferation of neonatal cardiomyocytes.

In summary, we demonstrated that TLR3 is necessary for the proliferation of neonatal cardiomyocytes and repair and regeneration of ischemic injured hearts. The mechanisms involve glycolysis dependent YAP1 activation via PP1a mediated suppression of MOB1 and LATS1 and through AMPK inactivation. Activated YAP1 increases the expression of miR-152 which targets DNMT1/ p27kip1, leading to cell proliferation (Fig. 9). Activation of TLR3 could be a novel strategy for the treatment of ischemic heart injury.

\section{Materials and Methods}

\section{Animals}

TLR3 deficient $\left(\right.$ TLR $^{-1-}$ ) and wild type C57BL/6 mice were obtained from Jackson Laboratory (Indianapolis, IN). The mice were maintained in the Division of Laboratory Animal Resources at East Tennessee State University. The experiments outlined in this manuscript conform to the Guide for the Care and Use of Laboratory Animals published by the National Institutes of Health (NIH Publication, $8^{\text {th }}$ Edition, 2011). The animal care and experimental protocols were approved by the ETSU Committee on Animal Care.

\section{Induction of Myocardial MI Injury}

1 day old (P1) mice were subjected to myocardial infarction (MI) as described previously [40]. Briefly, the neonatal mice were anesthetized by hypothermia on ice for 3-5 min. The chest was swabbed with betadine and opened at the fourth intercostal space. 8-0 silk ligature was used for LAD permanent ligation. The ribs were sutured together and the chest wall incision closed. The pups were warmed immediately after surgery by hand. Once all the surgeries were completed, the blood and skin of the pups were cleaned with mixed stuff from mother's cage before the pups were sent back to mother's cage. Cardiac function was assessed by echocardiography for up to 21 days after induction of MI as described previously [46, 47]. Ejection fraction (EF\%) and percent fractional shortening (FS\%) were calculated $[46,47]$.

\section{Isolation of Neonatal Cardiomyocytes}

Neonatal cardiomyocytes were isolated from 1 day old (P1) WT mouse hearts as described previously [48]. Briefly, hearts were harvested from P1 neonatal mice and cut into small pieces followed by digestion in dissociation buffer $(116 \mathrm{mM} \mathrm{NaCl}, 20 \mathrm{mM}$ Hepes, $0.8 \mathrm{mM}$ $\mathrm{Na}_{2} \mathrm{HPO}_{4}, 5.6 \mathrm{mM}$ glucose, $5.4 \mathrm{mM} \mathrm{KCl}$, and $0.8 \mathrm{mM}$ $\mathrm{MgSO}_{4}, \mathrm{pH} 7.35$ ) containing $0.6 \mathrm{mg} / \mathrm{mL}$ of pancreatin and $0.4 \mathrm{mg} / \mathrm{mL}$ Collagenase Type II for $10 \mathrm{~min}$. The supernatant was removed and digestion buffer was added. After several repeated digestions with $10 \mathrm{~min}$ for each step, the cell suspension was added with fetal bovine serum followed by centrifugation for $5 \mathrm{~min}$ at $100 \times g$. The cells were seeded onto uncoated dishes for $2-4 \mathrm{~h}$. The supernatant was recollected and plated into gelatin pre-coated plates. 


\section{Cardiomycoyte Proliferation}

The proliferation of neonatal cardiomyocytes was measured by EdU incorporation and anti-Ph3 staining [18, 19, 48]. EdU incorporation was examined by Click-iT EdU imaging kit (Life Technologies) according to the manufacture's protocol. Nuclei were stained with DAPI and cardiomyocytes were stained with a monoclonal anti-actin (Abcam, Cambridge, MA). The proliferation rate was calculated by dividing $\mathrm{EdU}^{+}$cardiomyocytes by the total number of cardiomyocytes $[18,19,48]$ in a blinded fashion. The experiments were performed at least three times (experimental replicates) and each experimental replicate contained one-two biological replicates.

\section{qPCR Assay}

mRNAs and microRNAs were isolated from heart tissues or cultured cells using the miRNAs isolation kit (RNAzol@RT, MRC) in accordance with the manufacture's protocol. Quantitative real-time (qPCR) was conducted using a 4800 Real-time PCR machine (Bio-Rad). mRNAs and microRNAs levels were quantified by qPCR using specific Taqman assays (Applied Biosystems, USA) and Taqman Universal Master Mix (Applied Biosystems). The levels of miR-152 and mRNA levels of YAP1 or TAZ were quantified with the $2(-\Delta \Delta c t)$ relative quantification method that was normalized to the snRU6 or $\beta$-Actin (Applied Biosystems).

\section{In Vitro Experiments}

The isolated neonatal cardiomyocytes were maintained in Dulbecco's modified Eagle's medium (DMEM) supplemented with $10 \%$ fetal bovine serum under $5 \% \mathrm{CO}_{2}$ at $37^{\circ}$ C. To determine the role of TLR3 in the metabolism and proliferation of neonatal cardiomyocytes, the cells were treated with TLR3 specific ligand, Poly (I:C) $(1 \mu \mathrm{g} / \mathrm{ml})$. Cell proliferation was assessed by EdU incorporation (10 $\mu \mathrm{mol} / \mathrm{L}$ ) [48]. The glycolytic capacity (ECAR) was examined with a Seahorse system. To examine the role of glycolysis and protein phosphotase 1 (PP1a) in TLR3mediated YAP1 activation and cardiomyocyte proliferation, the isolated cardiomyocytes were treated with the glycolytic inhibitor, 2-DG $(5 \mathrm{mmol} / \mathrm{L})$ or PP1a inhibitor, okadaic acid, $(0.5 \mathrm{mmol} / \mathrm{L})$ respectively, before the cells were treated with Poly (I:C) at $1 \mu \mathrm{g} / \mathrm{mL}$. To determine whether YAP1 or miR-152 is necessary for TLR3mediated cardiomyocyte proliferation, the isolated cardiomyocytes were treated with YAP1 inhibitor verteporfin (VP, $1 \mathrm{mmol} / \mathrm{L})$ or transfected with siRNA-Con $(80 \mathrm{nmol} /$ L), siRNA-YAP1 $(80 \mathrm{nmol} / \mathrm{L})$, microRNA-Con mimics (40 nmol/L), miR-152 mimics (40 nmol/L), anti-miR-Con mimics $(60 \mathrm{nmol} / \mathrm{L})$, anti-miR-152 mimics $(60 \mathrm{nmol} / \mathrm{L})$, respectively prior to Poly (I:C) treatment. To determine whether AMPK was involved in TLR3-mediated YAP1 activation and cardiomyocyte proliferation, the AMPK activator Metformin $(1 \mathrm{mmol} / \mathrm{L})$ was used prior to Poly (I: C) treatment. The experiments were performed at least three times (experimental replicates) and each experimental replicate contained one-two biological replicates.

\section{AAV Virus Packaging}

pAAV.cTnT::3Flag-hYAP and pAAV.cTnT::Luciferase vectors were kindly provided by Dr. William T. Pu (Harvard Stem Cell Institute). AAV virus was packaged in293T cells using AAV-DJ Helper Free Packaging System (Cell Biolabs, Inc). The hYAP vector contains an S-127-A mutation resulting in a constitutive active form. The AAV virus was purified and concentrated by $\mathrm{CsCl}$ gradient centrifugation. The AAV virus titer was determined by AAVpro Titration Kit (Takara).

\section{Immunoprecipitation}

Approximately $200 \mu \mathrm{g}$ of cellular proteins were subjected to immunoprecipitation with $2 \mu \mathrm{g}$ of antibody to PP1a (Santa Cruz Biotechnology, CA) followed by the addition of $15 \mu \mathrm{L}$ of protein A-agarose beads (Santa Cruz Biotechnology) as previously described [49]. The precipitates were washed four times with lysis buffer and subjected to immunoblotting with the appropriate antibodies.

\section{Immunoblotting}

Immunoblotting was performed as described previously $[46,47]$. The primary antibodies (p-LATS1, LATS1, pYAP1 (S127), p-YAP1 (S397), YAP1, p-MOB1, MOB1, p-AMPK, AMPK, p27kip1, DNMT1) and peroxidaseconjugated secondary antibody were purchased from Cell Signaling Technology, Inc. The PP1a antibody was purchased from Santa Cruz Biotechnology. The signals were quantified using the G:Box gel imaging system by Syngene (Syngene, Fredric, MD).

\section{Statistical Analysis}

Data were expressed as mean $\pm \mathrm{SD}$. Comparisons of data between groups were made using one-way analysis of variance (ANOVA), and Tukey's procedure for multiplerange tests was performed. $P<0.05$ was considered to be significant.

Acknowledgements We thank William T. Pu (Harvard Stem Cell Institute) for providing pAAV.cTnT::3Flag-hYAP and pAAV.cTnT:: 
Luciferase vectors, and Gary Wright and Ying Li (ETSU) for technical assistance in the glycolytic capacity (ECAR) examination with the Seahorse system. This work was supported, in part, by NIH HL071837 (Li), GM083016 (Li and Williams), GM53522 (Williams), GM119197 (Williams) and C06RR0306551 to ETSU.

Author contributions XHW and CFL conceived the project, designed the experiments, analyzed and interpreted data, and wrote the manuscript. XHW, TZH and YPH performed experiments. LL, RK and DW contributed to the scientific discussion and data interpretation. JK provided support for statistical analysis.

\section{Compliance with ethical standards}

Conflict of Interest The authors declare that there are no competing financial interests.

Open Access This article is licensed under a Creative Commons Attribution-NonCommercial-NoDerivatives 4.0 International License, which permits any non-commercial use, sharing, distribution and reproduction in any medium or format, as long as you give appropriate credit to the original author(s) and the source, and provide a link to the Creative Commons license. You do not have permission under this license to share adapted material derived from this article or parts of it. The images or other third party material in this article are included in the article's Creative Commons license, unless indicated otherwise in a credit line to the material. If material is not included in the article's Creative Commons license and your intended use is not permitted by statutory regulation or exceeds the permitted use, you will need to obtain permission directly from the copyright holder. To view a copy of this license, visit http://creativecommons.org/licenses/by-nc-nd/4.0/.

\section{References}

1. Go AS, Mozaffarian D, Roger VL, Benjamin EJ, Berry JD, Blaha MJ, et al. Heart disease and stroke statistics--2014 update: a report from the American Heart Association. Circulation 2014;129: e28-e292.

2. Porrello ER, Mahmoud AI, Simpson E, Hill JA, Richardson JA, Olson EN, et al. Transient regenerative potential of the neonatal mouse heart. Science 2011;331:1078-80.

3. Poss KD, Wilson LG, Keating MT. Heart regeneration in zebrafish. Science 2002;298:2188-90.

4. Bryant DM, O'Meara CC, Ho NN, Gannon J, Cai L, Lee RT. A systematic analysis of neonatal mouse heart regeneration after apical resection. J Mol Cell Cardiol 2014;79:315-18.

5. Harris DA, Das AM. Control of mitochondrial ATP synthesis in the heart. Biochem J 1991;280(Pt 3):561-73.

6. Yutzey KE, Cardiovascular biology: Switched at birth. Nature. 2014;509:572-3.

7. Puente BN, Kimura W, Muralidhar SA, Moon J, Amatruda JF, Phelps KL, et al. The oxygen-rich postnatal environment induces cardiomyocyte cell-cycle arrest through DNA damage response. Cell. 2014;157:565-79.

8. Nie B, Wang H, Laurent T, Ding S. Cellular reprogramming: a small molecule perspective. Curr Opin Cell Biol. 2012;24: 784-92.

9. Panopoulos AD, Yanes O, Ruiz S, Kida YS, Diep D, Tautenhahn $\mathrm{R}$, et al. The metabolome of induced pluripotent stem cells reveals metabolic changes occurring in somatic cell reprogramming. Cell Res. 2014;22:168-77.

10. Zhang J, Nuebel E, Daley GQ, Koehler CM, Teitell MA. Metabolic regulation in pluripotent stem cells during reprogramming and self-renewal. Cell Stem Cell 2012;11:589-95.
11. Medzhitov R, Preston-Hurlburt P, Janeway CA Jr.. A human homologue of the Drosophila Toll protein signals activation of adaptive immunity. Nature 1997;388:394-7.

12. Aderem A, Ulevitch RJ. Toll-like receptors in the induction of the innate immune response. Nature 2000;406:782-7.

13. Cheng SC, Quintin J, Cramer RA, Shepardson KM, Saeed S, Kumar $\mathrm{V}$, et al. mTOR- and HIF-1alpha-mediated aerobic glycolysis as metabolic basis for trained immunity. Science 2014;345:1250684.

14. Everts B, Amiel E, Huang SC, Smith AM, Chang CH, Lam WY, et al. TLR-driven early glycolytic reprogramming via the kinases TBK1-IKKvarepsilon supports the anabolic demands of dendritic cell activation. Nat Immunol 2014;15:323-32.

15. O'Neill LA. Glycolytic reprogramming by TLRs in dendritic cells. Nat Immunol 2014;15:314-5.

16. Yu FX, Zhao B, Guan KL. Hippo pathway in organ size control, tissue homeostasis, and cancer. Cell 2015;163:811-28.

17. Zhou Q, Li L, Zhao B, Guan KL. The hippo pathway in heart development, regeneration, and diseases. Circ Res 2015;116:1431-47.

18. von GA, Lin Z, Schlegelmilch K, Honor LB, Pan GM, Buck JN, et al. YAP1, the nuclear target of Hippo signaling, stimulates heart growth through cardiomyocyte proliferation but not hypertrophy. Proc Natl Acad Sci USA 2012;109:2394-9.

19. Lin Z, Zhou P, von GA, Gu F, Ma Q, Chen J, et al. Pi3kcb Links Hippo-YAP and PI3K-AKT Signaling Pathways to Promote Cardiomyocyte Proliferation and Survival. Circ Res 2015;116:35-45.

20. Heallen T, Morikawa Y, Leach J, Tao G, Willerson JT, Johnson $\mathrm{RL}$, et al. Hippo signaling impedes adult heart regeneration. Development 2013;140:4683-90.

21. Xin M, Kim Y, Sutherland LB, Murakami M, Qi X, McAnally J, et al. Hippo pathway effector Yap promotes cardiac regeneration. Proc Natl Acad Sci USA 2013;110:13839-44.

22. Tian Y, Liu Y, Wang T, Zhou N, Kong J, Chen L, et al. A microRNA-Hippo pathway that promotes cardiomyocyte proliferation and cardiac regeneration in mice. Sci Transl Med 2015;7:279ra38.

23. Zhao B, Li L, Tumaneng K, Wang CY, Guan KL. A coordinated phosphorylation by Lats and CK1 regulates YAP stability through SCF(beta-TRCP). Genes Dev 2010;24:72-85.

24. Hergovich A. MOB control: reviewing a conserved family of kinase regulators. Cell Signal 2011;23:1433-40.

25. Lv XB, Liu CY, Wang Z, Sun YP, Xiong Y, Lei QY, et al. PARD3 induces TAZ activation and cell growth by promoting LATS1 and PP1 interaction. Embo Rep 2015;16:975-85.

26. Liu CY, Lv X, Li T, Xu Y, Zhou X, Zhao S, et al. PP1 cooperates with ASPP2 to dephosphorylate and activate TAZ. J Biol Chem 2011;286:5558-66.

27. Arad M, Seidman CE, Seidman JG. AMP-activated protein kinase in the heart: role during health and disease. Circ Res 2007;100:474-88.

28. Mo JS, Meng Z, Kim YC, Park HW, Hansen CG, Kim S, et al. Cellular energy stress induces AMPK-mediated regulation of YAP and the Hippo pathway. Nat Cell Biol 2015;17:500-10.

29. Guo SL, Peng Z, Yang X, Fan KJ, Ye H, Li ZH, et al. miR-148a promoted cell proliferation by targeting p27 in gastric cancer cells. Int J Biol Sci 2011;7:567-74.

30. Wang YS, Chou WW, Chen KC, Cheng HY, Lin RT, Juo SH. MicroRNA-152 mediates DNMT1-regulated DNA methylation in the estrogen receptor alpha gene. PLoS One 2012;7:e30635.

31. Sim CB, Ziemann M, Kaspi A, Harikrishnan KN, Ooi J, Khurana I, et al. Dynamic changes in the cardiac methylome during postnatal development. Faseb J 2015;29:1329-43.

32. Resnitzky D, Hengst L, Reed SI. Cyclin A-associated kinase activity is rate limiting for entrance into $\mathrm{S}$ phase and is negatively regulated in G1 by p27Kip1. Mol Cell Biol 1995;15:4347-52. 
33. Lu C, Ren D, Wang X, Ha T, Liu L, Lee EJ, et al. Toll-like receptor 3 plays a role in myocardial infarction and ischemia/ reperfusion injury. Biochim Biophys Acta 2014;1842:22-31.

34. Grote K, Schuett H, Salguero G, Grothusen C, Jagielska J, Drexler $\mathrm{H}$, et al. Toll-like receptor $2 / 6$ stimulation promotes angiogenesis via GM-CSF as a potential strategy for immune defense and tissue regeneration. Blood 2010;115:2543-52.

35. Lee J, Sayed N, Hunter A, Au KF, Wong WH, Mocarski ES, et al. Activation of innate immunity is required for efficient nuclear reprogramming. Cell 2012;151:547-58.

36. Sayed N, Wong WT, Ospino F, Meng S, Lee J, Jha A, et al. Transdifferentiation of Human Fibroblasts to Endothelial Cells: Role of Innate Immunity. Circulation 2015;131:300-309.

37. Bergmann O, Bhardwaj RD, Bernard S, Zdunek S, BarnabeHeider F, Walsh S, et al. Evidence for cardiomyocyte renewal in humans. Science 2009;324:98-102.

38. Ali SR, Hippenmeyer S, Saadat LV, Luo L, Weissman IL, Ardehali R. Existing cardiomyocytes generate cardiomyocytes at a low rate after birth in mice. Proc Natl Acad Sci USA 2014;111:8850-5.

39. Senyo SE, Steinhauser ML, Pizzimenti CL, Yang VK, Cai L, Wang M, et al. Mammalian heart renewal by pre-existing cardiomyocytes. Nature 2013;493:433-6.

40. Aurora AB, Porrello ER, Tan W, Mahmoud AI, Hill JA, BasselDuby $\mathrm{R}$, et al. Macrophages are required for neonatal heart regeneration. J Clin Invest 2014;124:1382-92.

41. Lai SL, Marin-Juez R, Moura PL, Kuenne C, Lai JKH, Tsedeke AT, et al. Reciprocal analyses in zebrafish and medaka reveal that harnessing the immune response promotes cardiac regeneration. Elife 2017;6:e25605.
42. Bassat E, Mutlak YE, Genzelinakh A, Shadrin IY, Baruch UK, Yifa $\mathrm{O}$, et al. The extracellular matrix protein agrin promotes heart regeneration in mice. Nature 2017;547:179-84.

43. Morikawa Y, Heallen T, Leach J, Xiao Y, Martin JF. Dystrophinglycoprotein complex sequesters Yap to inhibit cardiomyocyte proliferation. Nature 2017;547:227-31.

44. DeRan M, Yang J, Shen CH, Peters EC, Fitamant J, Chan P, et al. Energy stress regulates hippo-YAP signaling involving AMPKmediated regulation of angiomotin-like 1 protein. Cell Rep 2014;9:495-503.

45. Tumaneng K, Schlegelmilch K, Russell RC, Yimlamai D, Basnet $\mathrm{H}$, Mahadevan $\mathrm{N}$, et al. YAP mediates crosstalk between the Hippo and PI(3)K-TOR pathways by suppressing PTEN via miR29. Nat Cell Biol 2012;14:1322-9.

46. Wang X, Ha T, Liu L, Zou J, Zhang X, Kalbfleisch J, et al. Increased expression of microRNA-146a decreases myocardial ischaemia/reperfusion injury. Cardiovasc Res 2013;97: 432-42.

47. Wang X, Ha T, Zou J, Ren D, Liu L, Zhang X, et al. MicroRNA$125 \mathrm{~b}$ protects against myocardial ischaemia/reperfusion injury via targeting p53-mediated apoptotic signalling and TRAF6. Cardiovasc Res 2014;102:385-95.

48. Chen J, Huang ZP, Seok HY, Ding J, Kataoka M, Zhang Z, et al. mir-17-92 cluster is required for and sufficient to induce cardiomyocyte proliferation in postnatal and adult hearts. Circ Res 2013;112:1557-66.

49. Cao Z, Ren D, Ha T, Liu L, Wang X, Kalbfleisch J, et al. CpGODN, the TLR9 agonist, attenuates myocardial ischemia/reperfusion injury: involving activation of PI3K/Akt signaling. Biochim Biophys Acta 2013;1832:96-104. 